\title{
Initialization-free Distributed Algorithms for Optimal Resource Allocation with Feasibility Constraints and Application to Economic Dispatch of Power Systems st
}

\author{
Peng Yi ${ }^{\mathrm{a}}$, Yiguang Hong ${ }^{\mathrm{b}}$, Feng $\mathrm{Liu}^{\mathrm{c}}$ \\ ${ }^{a}$ Department of Electrical $\mathcal{E}$ Computer Engineering, University of Toronto \\ ${ }^{b}$ Key Lab of Systems and Control, Academy of Mathematics and Systems Science, Chinese Academy of Sciences, \\ ${ }^{c}$ Department of Electrical Engineering, Tsinghua University
}

\begin{abstract}
In this paper, the distributed resource allocation optimization problem is investigated. The allocation decisions are made to minimize the sum of all the agents' local objective functions while satisfying both the global network resource constraint and the local allocation feasibility constraints. Here the data corresponding to each agent in this separable optimization problem, such as the network resources, the local allocation feasibility constraint, and the local objective function, is only accessible to individual agent and cannot be shared with others, which renders new challenges in this distributed optimization problem. Based on either projection or differentiated projection, two classes of continuous-time algorithms are proposed to solve this distributed optimization problem in an initialization-free and scalable manner. Thus, no re-initialization is required even if the operation environment or network configuration is changed, making it possible to achieve a "plug-and-play" optimal operation of networked heterogeneous agents. The algorithm convergence is guaranteed for strictly convex objective functions, and the exponential convergence is proved for strongly convex functions without local constraints. Then the proposed algorithm is applied to the distributed economic dispatch problem in power grids, to demonstrate how it can achieve the global optimum in a scalable way, even when the generation cost, or system load, or network configuration, is changing.
\end{abstract}

Keywords: Resource allocation, Distributed optimization, Multi-agent system, Plug-and-play algorithm, Gradient flow, Projected dynamical system, Economic dispatch

\section{Introduction}

Resource allocation is one of the most important problems in network optimization, which has been widely investigated in various areas such as economics systems, communication networks, sensor networks, and power grids. The allocation 'decisions may be made centrally by gathering all the network data together to a decision-making center, and then sent back to corresponding agents (referring to Ibaraki \& Katoh (1988)). On the other hand, differing from this centralized policy, the master-slave-type decentralized algorithms, either price-based (Arrow \& Hurwicz (1960)) or resource-based (Heal (1969)), -are constructed to achieve the optimal allocations by the local computations in the slave agents under the coordinations of the master/center through a one-to-all communication architecture. However, these methods may not be suitable or effective for the resource allocation in large-scale networks with numerous

\footnotetext{
This paper was not presented at any IFAC meeting. This work is supported by Beijing Natural Science Foundation (4152057), NSFC (61333001, 61573344), and Program 973 (2014CB845301/2/3). This work is also partly supported by the National Natural Science Foundation of China (No. 51377092), Foundation of Chinese Scholarship Council (CSC No. 201506215034). Corresponding author: Yiguang Hong, Tel. +86(010)82541824. Fax +86(010)62587343.

Email addresses: peng.yi@utoronto.ca (Peng Yi), yghong@iss.ac.cn (Yiguang Hong), lfeng@mail.tsinghua.edu.cn (Feng Liu)
}

heterogeneous agents due to complicated network structures, heavy communication burden, privacy concerns, unbearable time delays, and unexpected single-point failures. Therefore, fully distributed resource allocation optimization algorithms are highly desirable.

Distributed optimization, which cooperatively achieves optimal decisions by the local manipulation with private data and the diffusion of local information through a multi-agent network, has drawn more and more research attention in recent years. To circumvent the requirement of control center or master, various distributed optimization models or algorithms have been developed (Nedic, Ozdaglar, \& Parrilo (2010), Sayed (2014), Lou. Shi. Johansson. \& Hong (2014), and Yi \& Hong (2014)). In light of the increasing attention to distributed optimization and the seminal work on distributed resource allocation in Ho, Servi. \& Suri (1980), some distributed algorithms for resource allocation optimization have been proposed in Xiao \& Boyd (2006), Lakshmanan \& Farias (2008), Necoara, Nesterov, \& Glineur (2011), Ghadimi, Shames, \& Johansson (2013), and Beck, Nedic, Ozdaglar. \& Teboulle (2014).

Continuous-time gradient flow algorithms have been widely investigated for convex optimization after the pioneer work Arrow. Huwicz, \& Uzawa (1958), and detailed references can be found in Liao, Qi, \& Qi (2004) and Bhaya \& Kaszkurewicz (2006). Gradient flow algorithms 
have been applied to network control and optimization ( Low, Paganini, \& Doyle (2002), Feijer \& Paganini (2010) and Ferragut \& Paganini (2014)), neural networks (Liao, Qi, \& Qi (2004)), and stochastic approximation (Dupuis \& Kushner (1987)). Recently, continuous-time gradient flow algorithms have been adopted for solving unconstrained distributed optimization problems (see Wang \& Elia (2011), Gharesifard \& Cortés (2014), Droge, Kawashima \& Egerstedt (2014), and Kia, Cortés, \& Martinez (2015)). Furthermore, the projection-based gradient flow dynamics have been employed for solving the complicated constrained optimization problems in Venets (1985), Nagurney \& Zhang (1995), Xia \& Wang (2000), Gao (2003) and Cherukuri, Mallada, \& Cortés (2016), and the projected gradient flow ideas began to be applied to distributed constrained optimization (see Liu \& Wang (2015), Oiu, Liu, \& Xie (2014) and Yi, Hong \& Liu (2015)).

The economic dispatch, one of the key concerns in power grids, is to find the optimal secure generation allocation to balance the system loads, and hence, can be regarded as a special resource allocation problem. In recent years, there has been increasing research attention in solving economic dispatch problems through a multi-agent system in a distributed manner to meet the ever growing challenges raised by increasing penetration of renewable energies and deregulation of power infrastructure (Cavraro, Carli, \& Zampieri (2014) and Zhang, Liu, Wang, Liu, \& Ferrese (2015)). Mathematically, this boils down to a particular distributed resource allocation optimization problem. Furthermore, there were various continuous-time algorithms for the Distributed Economic Dispatch Problem (DEDP). For example, Zhao, Topcu, Li, \& Low (2014) showed that the physical power grid dynamics could serve as a part of a primal-dual gradient flow algorithm to solve the DEDP, and in fact, it considered physical network interconnections and generator dynamics explicitly, providing a quite comprehensive method and inspiring insights. Moreover, Cherukuri, \& Cortés (2015) solved the DEDP by combining the penalty method and the distributed continuous-time algorithm in Ho, Servi, \& Suri (1980), and proposed a procedure to fulfill the initialization requirement, while Cherukuri \& Cortés (2014) constructed a novel initialization-free distributed algorithm to achieve DEDP given one agent knowing the total system loads.

Motivated by various practical problems, including the DEDP in power grids, we study a Distributed Resource Allocation Optimization (DRAO) problem, where each agent can only manipulate its private data, such as the local objective function, Local Feasibility Constraint (LFC), and local resource data. Such data in practice cannot be shared or known by other agents. As the total network resource is the sum of individual agent's local resources, the agents need to cooperatively achieve the optimal resource allocation in a distributed way, so that the global objective function (as the sum of all local objective functions) is minimized with all the constraints (including the network resource constraint and LFCs) satisfied. Note that the LFC is critical for the (secure) operation of practical networks (referring to the communication system in Johari \& Tsitsiklis (2004) and D'Amico, Sanguinetti \& Palomar (2014) as an example), even though it was not considered in most existing DRAO works. Particularly, for the DEDP in power grids, the generation of each generator must be limited within its box-like capacity bounds. The consideration of LFCs brings remarkable difficulties to existing distributed algorithms designed for the DRAO without LFCs, because the KKT (optimality) conditions for the DRAO with and without LFCs are totally different (referring to Remark 3.3). So far, many DEDP works (such as Zhao, Topcu, Li, \& Low (2014), Zhang, Liu, Wang, Liu, \& Ferrese (2015) and Cherukuri, \& Cortés (2015) and Cherukuri \& Cortés (2014)) have only considered the box-like LFCs. However, the requirement from power industries, such as the secure operation of inverter-based devices in smart grids, promotes the demand to deal with non-box LFCs. This extension is nontrivial, and we will show how to handle it systematically by using projected dynamics in this paper.

Another crucial albeit difficult problem is the initialization coordination among all agents. Many existing results are based on initialization coordination procedures to guarantee that the initial allocations satisfy the network resource constraint, which may only work well for static networks. However, for a dynamical network, the resource has to be re-allocated once the network configuration changes. Therefore, the initialization coordination has to be re-performed whenever these optimization algorithms re-start, which considerably degrades their applicability. Taking the DEDP as an example, the initialization needs to be coordinated among all agents whenever local load demand or generation capacity/cost changes, or any distributed generator plugs in or leaves off (see Cherukuri, \& Cortés (2015) for an initialization procedure). This issue has to be well addressed for achieving highly-flexible power grids with the integration of ever-increasing renewables.

The objective of this paper is to propose an initialization-free methodology to solve the DARO with local LFCs. The main technical contributions of this paper are highlighted as follows:

- By employing the (differentiated) projection operation, two fully distributed continuous-time algorithms are proposed as a kind of projected dynamics, with the local allocation of each agent kept within its own LFC set. Moreover, the algorithms ensure the network resource constraint asymptotically without requiring it being satisfied at the initial points. Therefore, it is initialization-free, different from those given in Xiao \& Boyd (2006), Lakshmanan \& Farias (2008), Necoara, Nesterov, \& Glineur (2011) and Ghadimi, Shames, \& Johansson (2013), and moreover, provides novel initialization-free algorithms different from the one given in Cherukuri \& Cortés (2014).

- The convergence of the two projected algorithms is shown by the properties of Laplacian matrix and projection operation as well as the LaSalle invariance principle. The result can be regarded as an extension of some existing distributed optimization algorithms (Wang \& Elia (2011), Kia, Cortés, \& Martinez (2015), Qiu, Liu, \& Xie (2014), and Liu \& Wang (2015)) and an application of projected 
dynamics for variational inequalities (Gao (2003) and Xia \& Wang (2000)) to the DRAO problem.

- The proposed algorithms can be directly applied to the DEDP in power systems considering generation capacity limitations. It enables the plug-and-play operation for power grids with high-penetration of flexible renewables. Our algorithms are essentially different from the ones provided in Cherukuri, \& Cortés (2015) and Cherukuri \& Cortés (2014), and address multi-dimensional decision variables and general non-box LFCs. Simulation results demonstrate that the algorithm effectively deals with various data and network configuration changes, and also illustrate the algorithm scalability.

The reminder of this paper is organized as follows. The preliminaries are given and then the DRAO with LFCs is formulated with the basic assumptions in Section 2. Then a distributed algorithm in the form of projected dynamics is proposed with its convergence analysis in Section 3. In Section 4, a differentiated projected algorithm is proposed with its convergence analysis for DRAO with strongly convex objective functions, and an exponential convergence rate is obtained in the case without LFCs. Moreover, the application to the DEDP in power systems is shown in section 5 with numerical experiments. Finally, the concluding remarks are given in Section 6.

Notations: Denote $\mathbf{R}_{\geq 0}$ as the set of nonnegative real numbers. Denote $\mathbf{1}_{m}=(1, \ldots, 1)^{T} \in \mathbf{R}^{m}$ and $\mathbf{0}_{m}=(0, \ldots, 0)^{T} \in \mathbf{R}^{m}$. Denote $\operatorname{col}\left(x_{1}, \ldots, x_{n}\right)=\left(x_{1}^{T}, \cdots, x_{n}^{T}\right)^{T}$ as the column vector stacked with vectors $x_{1}, \ldots, x_{n} . I_{n}$ denotes the identity matrix in $\mathbf{R}^{n \times n}$. For a matrix $A=\left[a_{i j}\right], a_{i j}$ or $A_{i j}$ stands for the matrix entry in the $i$ th row and $j$ th column of $A . A \otimes B$ denotes the Kronecker product of matrixes $A$ and $B$. Denote $\times_{i=1, \ldots, n} \Omega_{i}$ as the Cartesian product of the sets $\Omega_{i}, i=1, \ldots, n$. Denote the set of interiors of set $K$ as $\operatorname{int}(K)$, and the boundary set of set $K$ as $\partial K$.

\section{Preliminaries and problem formulation}

In this section, we first give the preliminary knowledge related to convex analysis and graph theory, and then formulate the DRAO problem of interest.

\subsection{Convex analysis and projection}

The following concepts and properties about convex functions, convex sets, and projection operations come from Ruszczynski (2006) and Bertsekas (2009). A differentiable convex function $f: \mathbf{R}^{m} \rightarrow \mathbf{R}$ has the locally Lipschitz continuous gradient, if, given any compact set $Q$, there is a constant $k^{Q}$ such that $\|\nabla f(x)-\nabla f(y)\| \leq k^{Q}\|x-y\|, \forall x, y \in Q$. A differentiable function $f(x)$ is called $\mu$-strongly convex on $\mathbf{R}^{m}$ if there exists a constant $\mu>0$ such that, for any $x, y \in \mathbf{R}^{m}$, $f(y) \geq f(x)+\nabla^{T} f(x)(y-x)+\frac{1}{2} \mu\|y-x\|^{2}$, or equivalently,

$$
(x-y)^{T}(\nabla f(x)-\nabla f(y)) \geq \mu\|x-y\|^{2} .
$$

The following notations describe the geometry properties of the convex set. Denote $C_{\Omega}(x)$ as the normal cone of $\Omega$ at $x$, that is, $C_{\Omega}(x)=\{v:\langle v, y-x\rangle \leq 0, \quad \forall y \in \Omega\}$. Define $c_{\Omega}(x)$ as $c_{\Omega}(x)=\{v:\|v\|=1,\langle v, y-x\rangle \leq 0, \forall y \in \Omega\}$ if $x \in \partial \Omega$, and $c_{\Omega}=\{\mathbf{0}\}$ if $x \in \operatorname{int}(\Omega)$. The feasible direction cone of $\Omega$ at $x$ is given as $K_{\Omega}(x)=\{d: d=\beta(y-x), y \in \Omega, \beta \geq 0\}$. The tangent cone of set $\Omega$ at $x$ is defined as $T_{\Omega}(x)=\{v: v=$ $\left.\lim _{k \rightarrow \infty} \frac{x^{k}-x}{\tau_{k}}, \tau_{k} \geq 0, \tau_{k} \rightarrow 0, x^{k} \in \Omega, x^{k} \rightarrow x\right\}$. Then $T_{\Omega}(x)$ is the closure of $K_{\Omega}(x)$ when $\Omega$ is a closed convex set, and is the polar cone to $C_{\Omega}(x)$, that is, $T_{\Omega}(x)=\left\{y:\langle y, d\rangle \leq 0, \forall d \in C_{\Omega}(x)\right\}$ (referring to Lemma 3.13 of Ruszczynski (2006)).

Define the projection of $x$ onto a closed convex set $\Omega$ by $P_{\Omega}(x)=\arg \min _{y \in \Omega}\|x-y\|$. The basic property of projection operation is

$$
\left\langle x-P_{\Omega}(x), P_{\Omega}(x)-y\right\rangle \geq 0, \forall x \in \mathbf{R}^{m}, \forall y \in \Omega .
$$

The following relationships can be derived from (2),

$$
\left\|x-P_{\Omega}(x)\right\|_{2}^{2}+\left\|P_{\Omega}(x)-y\right\|_{2}^{2} \leq\|x-y\|_{2}^{2}, \forall x \in \mathbf{R}^{m}, \forall y \in \Omega,
$$

and

$$
\left\|P_{\Omega}(x)-P_{\Omega}(y)\right\| \leq\|x-y\|, \forall x, y \in \mathbf{R}^{m} .
$$

The normal cone $C_{\Omega}(x)$ can also be defined as (Lemma 2.38 of Ruszczynski (2006))

$$
C_{\Omega}(x)=\left\{v: P_{\Omega}(x+v)=x\right\} .
$$

For a closed convex set $\Omega$, point $x \in \Omega$ and direction $v$, we define the differentiated projection operator as (Dupuis \& Kushner (1987) and Nagurney \& Zhang (1995)),

$$
\Pi_{\Omega}(x, v)=\lim _{\delta \rightarrow 0} \frac{P_{\Omega}(x+\delta v)-x}{\delta} .
$$

The basic properties of the differentiated projection operator are given as follows Brogliato, Daniilidis, Lemarchal, \& Acary (2006)).

Lemma 2.1. (i):If $x \in \operatorname{int}(\Omega)$, then $\Pi_{\Omega}(x, v)=v$; (ii): $x \in \partial \Omega$, and $\max _{n \in c_{\Omega}(x)}\langle v, n\rangle \leq 0$, then $\Pi_{\Omega}(x, v)=v$; (iii): $x \in \partial \Omega$, and $\max _{n \in c_{\Omega}(x)}\langle v, n\rangle \geq 0$, then $\Pi_{\Omega}(x, v)=v-\left\langle v, n^{*}\right\rangle n^{*}$, where $n^{*}=\arg \max _{n \in c_{\Omega}(x)}\langle v, n\rangle$. Therefore, the operator $\Pi_{\Omega}(x, v)$ in (6) is equivalent with the projection of $v$ onto $T_{\Omega}(x)$, i.e.,

$$
\Pi_{\Omega}(x, v)=P_{T_{\Omega}(x)}(v) .
$$

\subsection{Graph theory}

The following concepts of graph theory can be found in Mesbahi \& Egerstedt (2010). The information sharing or exchanging among the agents is described by graph $\mathcal{G}=(\mathcal{N}, \mathcal{E})$. The edge set $\mathcal{E} \subset \mathcal{N} \times \mathcal{N}$ contains all the information interactions. If agent $i$ can get information from agent $j$, then $(j, i) \in \mathcal{E}$ and agent $j$ belongs to agent $i$ 's neighbor set $\mathcal{N}_{i}=\{j \mid(j, i) \in \mathcal{E}\}$. $\mathcal{G}$ is said to be undirected when $(i, j) \in \mathcal{E}$ if and only if $(j, i) \in \mathcal{E}$. A path of graph $\mathcal{G}$ is a sequence of distinct agents in $\mathcal{N}$ such that any consecutive agents in the sequence corresponding to an edge of graph $\mathcal{G}$. Agent $j$ is said to be connected to agent $i$ if 
there is a path from $j$ to $i . \mathcal{G}$ is said to be connected if any two agents are connected.

Define adjacency matrix $A=\left[a_{i j}\right]$ of $\mathcal{G}$ with $a_{i j}=1$ if $j \in$ $\mathcal{N}_{i}$ and $a_{i j}=0$ otherwise. Define the degree matrix Deg $=$ $\operatorname{diag}\left\{\sum_{j=1}^{n} a_{1 j}, \ldots, \sum_{j=1}^{n} a_{n j}\right\}$. Then the Laplacian of graph $\mathcal{G}$ is $L=D e g-A$. When $\mathcal{G}$ is a connected undirected graph, 0 is a simple eigenvalue of Laplacian $L$ with the eigenspace $\left\{\alpha \mathbf{1}_{n} \mid \alpha \in\right.$ $\mathbf{R}\}$, and $L \mathbf{1}_{n}=\mathbf{0}_{n}, \mathbf{1}_{n}^{T} L=\mathbf{0}_{n}^{T}$, while all other eigenvalues are positive. Denote the eigenvalues of $L$ in an ascending order as $0<s_{2} \leq \cdots \leq s_{n}$. Then, by the Courant-Fischer Theorem,

$$
\min _{\substack{x \neq 0 \\ \mathbf{1}^{T} x=0}} x^{T} L x=s_{2}\|x\|_{2}^{2}, \quad \max _{x \neq \mathbf{0}} x^{T} L x=s_{n}\|x\|_{2}^{2} .
$$

\subsection{Problem formulation}

Consider a group of agents with the index set $\mathcal{N}=\{1, \ldots, n\}$ to make an optimal allocation of network resource under both the network resource constraint and LFCs. Agent $i$ can decide its local allocation $x_{i} \in \mathbf{R}^{m}$, and can access the local resource data $d_{i} \in \mathbf{R}^{m}$. The total network resource is $\sum_{i \in \mathcal{N}} d_{i}$, and therefore, the allocation should satisfy the network resource constraint: $\sum_{i \in \mathcal{N}} x_{i}=\sum_{i \in \mathcal{N}} d_{i}$. Furthermore, the allocation of agent $i$ should satisfy the local feasibility constraint (LFC): $x_{i} \in \Omega_{i}$, where $\Omega_{i} \subset \mathbf{R}^{m}$ is a closed convex set only known by agent $i$. Agent $i$ also has a local objective function $f_{i}\left(x_{i}\right): \mathbf{R}^{m} \rightarrow \mathbf{R}$ associated with its local allocation $x_{i}$. Denote $X=\operatorname{col}\left(x_{1}, \ldots, x_{n}\right) \in \mathbf{R}^{m n}$ as the allocation vector of the whole network. Then the task for the agents is to collectively find the optimal allocation corresponding to the DRAO problem as follows:

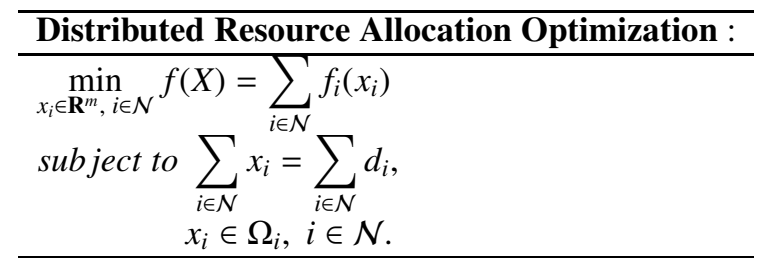

Clearly, problem (8) is an extension of the previous optimization models in Xiao \& Boyd (2006), Lakshmanan \& Farias (2008) and Necoara, Nesterov, \& Glineur (2011) by introducing the additional LFCs, that is, $x_{i} \in \Omega_{i}$. Clearly, $x_{i} \in \Omega_{i}$ also generalizes previous box constraints in Johari \& Tsitsiklis (2004), D’Amico, Sanguinetti \& Palomar (2014) and Cherukuri \& Cortés (2014).

The following assumptions are given for (8), which were also adopted for the distributed optimization or resource allocation in Feijer \& Paganini (2010), Kia, Cortés, \& Martinez (2015), and Liu \& Wang (2015).

Assumption 1. The functions $f_{i}\left(x_{i}\right), i \in \mathcal{N}$ are continuously differentiable convex functions with locally Lipschitz continuous gradients and positive definite Hessians over $\mathbf{R}^{m}$.

Assumption 1 implies that $f_{i}\left(x_{i}\right)$ 's are strictly convex, and hence guarantees the uniqueness of the optimal solution to (8).

Assumption 2. There exists a finite optimal solution $X^{*}$ to problem (8). The Slater's constraint condition is satisfied for DRAO (8), namely, there exists $\tilde{x}_{i} \in \operatorname{int}\left(\Omega_{i}\right), \forall i \in \mathcal{N}$, such that $\sum_{i \in \mathcal{N}} \tilde{x}_{i}=\sum_{i \in \mathcal{N}} d_{i}$.
Remark 2.2. Define the recession cone of a convex set $\Omega$ as $R_{\Omega}=\{d: x+\alpha d \in \Omega, \forall \alpha \geq 0, \forall x \in \Omega\}$. Then the sufficient and necessary condition for the existence of finite optimal solution to (8) is (referring to Proposition 3.2.2 of Bertsekas (2009))

$$
\times_{i \in \mathcal{N}} R_{\Omega_{i}} \cap\left\{\operatorname{Null}\left(\mathbf{1}_{n}^{T} \otimes I_{m}\right)\right\} \cap \times_{i \in \mathcal{N}} R_{f_{i}}=\mathbf{0},
$$

where $R_{\Omega_{i}}$ is the recession cone of $\Omega_{i}$ and $R_{f_{i}}$ is the recession cone of any nonempty level set of $f_{i}\left(x_{i}\right):\left\{x_{i} \in \mathbf{R}^{m} \mid f_{i}\left(x_{i}\right) \leq \gamma\right\}$.

In many practical cases, we have $R_{f_{i}}=\mathbf{0}$ (taking the quadratic function as an example). Furthermore, $R_{\Omega_{i}}=\mathbf{0}$ when $\Omega_{i}$ is compact. Therefore, the existence of a finite solution can be easily guaranteed and verified in many practical problems.

The local objective function $f_{i}\left(x_{i}\right)$, resource data $d_{i}$ and LFC set $\Omega_{i}$ are the private data for agent $i$, which are not shared with other agents. This makes (8) a distributed optimization problem. To fulfill the cooperations between agents for solving (8), the agents have to share their local information through a network $\mathcal{G}=(\mathcal{N}, \mathcal{E})$. Next follows an assumption about the connectivity of $\mathcal{G}$ to guarantee that any agent's information can reach any other agents, which is also quite standard for distributed optimization (Liu \& Wang (2015)).

Assumption 3. The information sharing graph $\mathcal{G}=(\mathcal{N}, \mathcal{E})$ is undirected and connected.

In a sum, the task of this paper is to design fully distributed algorithms for the agents to cooperatively find the optimal resource allocation to (8) without any center. In other words, agent $i$ needs to find its optimal allocation $x_{i}^{*}$ by manipulating its local private data $d_{i}, \Omega_{i}$, and $f_{i}\left(x_{i}\right)$ and by cooperations with its neighbor agents through $\mathcal{G}$.

\section{Projected algorithm for DRAO}

In this section, a distributed algorithm for (8) based on projected dynamics is proposed and analyzed. The distributed algorithm for agent $i$ is given as follows:

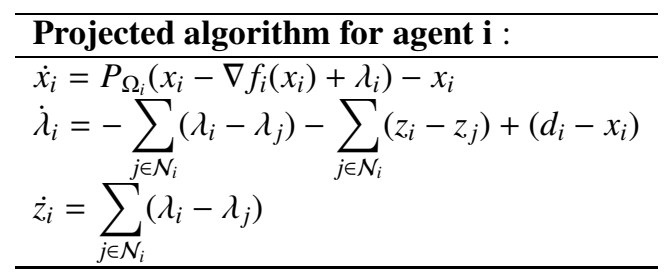

In algorithm (9), $x_{i} \in \mathbf{R}^{m}$ is the local allocation of agent $i$, and $\lambda_{i}, z_{i} \in \mathbf{R}^{m}$ are two auxiliary variables of agent $i$. Note that the algorithm (9) is fully distributed because agent $i$ only needs the local data (including $d_{i}, f_{i}\left(x_{i}\right)$, and $\left.\Omega_{i}\right)$ and the shared information $\left\{\lambda_{j}, z_{j}, j \in \mathcal{N}_{i}\right\}$ from its neighbor agents. Thereby, (9) does not need any center to handle all the data or coordinate all the agents. With the distributed algorithm (9), each agent has the autonomy and authority to formulate its own objective function and feasibility set, and hence, the privacy is kept within each agent. Because each agent can instantaneously react to 
its local data changes, it can quickly adapt its local decision. Therefore, the algorithm can be easily applied to large-scale networks.

The algorithm (9) can be understood based on the following observations. The duality of (8) with multiplier $\lambda \in \mathbf{R}^{m}$ is

$$
\max _{\lambda \in \mathbf{R}^{m}} q(\lambda)=\sum_{i \in \mathcal{N}} q_{i}(\lambda)=\sum_{i \in \mathcal{N}} \inf _{x_{i} \in \Omega_{i}}\left\{f_{i}\left(x_{i}\right)-\lambda^{T} x_{i}+\lambda^{T} d_{i}\right\} .
$$

Although some existing distributed algorithms in Nedic, Ozdaglar, \& Parrilo (2010) and Sayed (2014) addressed the dual problem (10), they need to solve a subproblem at each time (iteration) to calculate the gradients. In other words, two "time scales" are needed if applying existing distributed algorithms to (8). Here we aim to develop a simple algorithm without solving any subproblems. To this end, we formulate a constrained optimization problem with Laplacian matrix $L$ and $\Lambda=\operatorname{col}\left(\lambda_{1}, \ldots, \lambda_{n}\right) \in \mathbf{R}^{m n}$ as

$$
\begin{array}{ll}
\max _{\Lambda=\left(\lambda_{1}, \ldots, \lambda_{n}\right)} & Q(\Lambda)=\sum_{i \in \mathcal{N}} q_{i}\left(\lambda_{i}\right) \\
\text { subject to } & \left(L \otimes I_{m}\right) \Lambda=\mathbf{0}_{m n} .
\end{array}
$$

The augmented Lagrangian duality of 11 with multipliers $Z=$ $\operatorname{col}\left(z_{1}, \ldots, z_{n}\right) \in \mathbf{R}^{m n}$ is

$$
\min _{Z} \max _{\Lambda} Q(\Lambda, Z)=\sum_{i \in \mathcal{N}} q_{i}\left(\lambda_{i}\right)-Z^{T}\left(L \otimes I_{m}\right) \Lambda-\frac{1}{2} \Lambda^{T}\left(L \otimes I_{m}\right) \Lambda .
$$

Then the projected dynamics (9) is derived by applying the gradient flow to (10) and (12) along with the projection operation to guarantee the feasibility of LFCs.

From the KKT condition, we can show that the equilibrium point of (9) yields the optimal solution to problem (8). Denote $X=\operatorname{col}\left(x_{1}, \ldots, x_{n}\right), \nabla f(X)=\operatorname{col}\left(\nabla f_{1}\left(x_{1}\right), \cdots, \nabla f_{n}\left(x_{n}\right)\right)$, $D=\operatorname{col}\left(d_{1}, \cdots, d_{n}\right)$, and $\Omega=\times_{i \in \mathcal{N}} \Omega_{i}$. Write algorithm (9) in a compact form as

$$
\begin{aligned}
\dot{X} & =P_{\Omega}(X-\nabla f(X)+\Lambda)-X, \\
\dot{\Lambda} & =-\left(L \otimes I_{m}\right) \Lambda-\left(L \otimes I_{m}\right) Z+(D-X), \\
\dot{Z} & =\left(L \otimes I_{m}\right) \Lambda .
\end{aligned}
$$

Theorem 3.1. Under Assumptions 13 if the initial point $x_{i}(0) \in \Omega_{i}, \forall i \in \mathcal{N}$, then $x_{i}(t) \in \Omega_{i}, \forall t \geq 0, \forall i \in \mathcal{N}$, and $\operatorname{col}\left(X^{*}, \Lambda^{*}, Z^{*}\right)$ is the equilibrium point of the distributed algorithm (9) with $X^{*}$ as the optimal solution to (8).

Proof: Note that $\dot{x}_{i} \in T_{\Omega_{i}}\left(x_{i}\right), \forall x_{i} \in \Omega_{i}$ because $P_{\Omega_{i}}\left(x_{i}-\right.$ $\left.\nabla f_{i}\left(x_{i}\right)+\lambda_{i}\right) \in \Omega_{i}$. Given the initial point $x_{i}(0) \in \Omega_{i}$, by Nagumo's theorem (referring to page 174 and page 214 of Aubin \& Cellina (1984)), $x_{i}(t) \in \Omega_{i}, \forall t \geq 0$ (Related proofs can also be found in Liao, Qi, \& Qi (2004)).

To obtain the equilibrium point, we set $\dot{Z}=\mathbf{0}_{m n}$ and get $\Lambda^{*}=$ $\mathbf{1}_{n} \otimes \lambda^{*}, \lambda^{*} \in \mathbf{R}^{m}$, because the graph $\mathcal{G}$ is connected. $\dot{\Lambda}=\mathbf{0}_{m n}$ implies that $\left(L \otimes I_{m}\right) Z^{*}=D-X^{*}$. Because the graph $\mathcal{G}$ is undirected, $\mathbf{1}_{n}^{T} L=\mathbf{0}_{n}^{T}$ and $\left(\mathbf{1}_{n}^{T} \otimes I_{m}\right)\left(L \otimes I_{m}\right) Z=\left(\mathbf{1}_{n}^{T} L\right) \otimes I_{m} Z=$ $\left(\mathbf{1}_{n}^{T} \otimes I_{m}\right)(D-X)=\mathbf{0}_{m}$. Hence, $\sum_{i \in \mathcal{N}} d_{i}=\sum_{i \in \mathcal{N}} x_{i}^{*}$. Then, at the equilibrium point,

$$
\Lambda^{*}=\mathbf{1}_{n} \otimes \lambda^{*}, \lambda^{*} \in \mathbf{R}^{m},\left(L \otimes I_{m}\right) Z^{*}=D-X^{*}, \sum_{i \in \mathcal{N}} d_{i}=\sum_{i \in \mathcal{N}} x_{i}^{*} .
$$

Also, at the equilibrium point, $\dot{x}_{i}=0$ implies that $P_{\Omega_{i}}\left(x_{i}^{*}-\right.$ $\left.\nabla f_{i}\left(x_{i}^{*}\right)+\lambda^{*}\right)=x_{i}^{*}$. It follows from Lemma 2.38 of Ruszczynski (2006) that

$$
-\nabla f_{i}\left(x_{i}^{*}\right)+\lambda^{*} \in C_{\Omega_{i}}\left(x_{i}^{*}\right), i=1, \ldots, n .
$$
fies

Therefore, the equilibrium point $\operatorname{col}\left(X^{*}, \Lambda^{*}, Z^{*}\right)$ of (9) satis-

$$
\begin{aligned}
& \mathbf{0}_{m n} \in \nabla f\left(X^{*}\right)-\mathbf{1}_{n} \otimes \lambda^{*}+C_{\Omega}\left(X^{*}\right), \\
& \left(\mathbf{1}_{n}^{T} \otimes I_{m}\right) X^{*}=\left(\mathbf{1}_{n}^{T} \otimes I_{m}\right) D, \quad X^{*} \in \Omega,
\end{aligned}
$$

which is exactly the optimality condition (KKT) for DRAO (8) by Theorem 3.34 in Ruszczynski (2006). Thus, the conclusion follows.

Remark 3.2. It can be shown that the equilibrium point of (9) has $\lambda^{*}$ as the dual optimal solution to problem (10), and $Z^{*}$ as the dual optimal solution to problem (11), following a similar analysis routine of Theorem 3.1 and Proposition 5.3.2 in Bertsekas (2009). It can also be shown that any $\operatorname{col}\left(X^{*}, \mathbf{1}_{m} \otimes\right.$ $\left.\lambda^{*}, Z^{*}\right)$, with $X^{*}$ as the optimal solution to (8), $\lambda^{*}$ as the dual optimal solution to (10) and $Z^{*}$ as the dual optimal solution to (11), corresponds to an equilibrium point of (9). We do not discuss their details here for space limitations.

Remark 3.3. The differences between our work and some existing ones are listed as follows:

- The KKT condition for DRAO without LFC is

$$
\nabla f\left(X^{*}\right)=\mathbf{1}_{n} \otimes \lambda^{*},\left(\mathbf{1}_{n}^{T} \otimes I_{m}\right) X^{*}=\left(\mathbf{1}_{n}^{T} \otimes I_{m}\right) D .
$$

The KKT condition (14) for DRAO with LFC and the condition (15) for DRAO without LFC are totally different. (15) requires the optimal allocations to be the points satisfying the network resource constraint with the same marginal costs (gradients), while (14) requires the optimal allocations to be feasible in both network resource constraint and LFCs. The optimal allocations in (14) should also satisfy a variational inequality related to both the objective functions' gradients and the normal cones of the LFC sets. In fact, the marginal costs (gradients) at the optimal allocations of (14) do not necessarily reach the same levels, and the differences can be seen as the "price of allocation feasibility".

- The previous algorithms (except the one in Cherukuri \& Cortés (2014)) kept the network resource constraint satisfied (ensured its eventual feasibility) by setting feasible initial points through the initialization coordination procedure. In other words, the network resource constraint can be guaranteed only if it is satisfied at the initial moment. However, the initialization for the network resource constraint is quite restrictive for large-scale dynamical networks because it involves global coordination and has to be performed every time the network data/configuration changes. Moreover, it is not trivial to achieve the initialization coordination with both the LFCs and the network resource constraint (refer to Cherukuri, E Cortés (2015) for an initialization procedure with one dimensional interval constraint). 
- In fact, proportional-integral (PI)-type consensus dynamics (Wang E Elia (2011) and Gharesifard E Cortés (2014)) and projected gradient flows (Liu E Wang (2015)), which both have been utilized for distributed optimization, are combined together to obtain the algorithm (9) for the KKT condition (14). Local $\lambda_{i}$ acts as the local shadow price, and all the local shadow prices must reach consensus to be the global market clearing price. Therefore, a second-order PI-based consensus dynamics is incorporated into (9) with the integral variable $z_{i}$ summing up the disagreements between $\lambda_{i}$ and $\left\{\lambda_{j}, j \in \mathcal{N}_{i}\right\}$. Meanwhile, the dynamics of $x_{i}$ adjusts the local allocation by comparing the local shadow price and the local gradient, and also utilizes the projection operation in order to make the local allocation flowing within its LFC set all the time.

Therefore, the algorithms given in Ho, Servi, \& Suri (1980), Lakshmanan \& Farias (2008), Necoara, Nesterov, \& Glineur (2011) and Ghadimi, Shames, \& Johansson (2013) failed to solve (8) because they cannot ensure the LFCs given in (14). Note that the algorithm (9) can ensure LFCs even during the algorithm flow with projection operations. It only requires that each agent has its initial allocation belonging to its LFC set, which can be trivially accomplished by each agent with onestep local projection operation. Furthermore, algorithm (9) ensures the network resource constraint asymptotically without caring about whether it is satisfied at the initial points, and therefore, is free of initialization coordination procedure. Due to free of any center and initialization, algorithm (9) can adaptively handle online data without re-initialization whenever the network data/configuration changes. Moreover, it can work in a "plug-and-play" manner for dynamical networks with leavingoff or plugging-in of agents.

Next, let us analyze the convergence of (9), The analysis techniques are inspired by the projected dynamical systems for variational inequalities (referring to Xia \& Wang (2000) and Gao (2003)) and distributed optimization (referring to Shi, Johanssan, \& Hong (2013), Kia, Cortés, \& Martinez (2015), Oiu, Liu, \& Xie (2014) and Liu \& Wang (2015)).

Theorem 3.4. Under Assumptions [1,3] and given bounded initial points $x_{i}(0) \in \Omega_{i}, \forall i \in \mathcal{N}$, the trajectories of the algorithm (9) are bounded and converge to an equilibrium point of (9), namely, agent $i$ asymptotically achieves its optimal allocation $x_{i}^{*}$ of (8) with (9).

Proof: Take $m=1$ without loss of generality. Denote $\bar{\Omega}=$ $\Omega \times \mathbf{R}^{n} \times \mathbf{R}^{n}$. Define a new vector $S=\operatorname{col}(X, \Lambda, Z)$ and the vector function $F(S): R^{3 n} \rightarrow R^{3 n}$ as

$$
F(S)=\left(\begin{array}{c}
\nabla f(X)-\Lambda \\
L \Lambda+L Z-(D-X) \\
-L \Lambda
\end{array}\right)
$$

Recalling the form in (13) and the fact that $P_{\mathbf{R}^{n}}(x)=x, \forall x \in$ $\mathbf{R}^{n}$, the dynamics of all the agents can be written as

$$
\dot{S}=P_{\bar{\Omega}}(S-F(S))-S .
$$

Define $H(S)=P_{\bar{\Omega}}(S-F(S))$, and then give a Lyapunov function as

$$
V_{g}=-\langle F(S), H(S)-S\rangle-\frac{1}{2}\|H(S)-S\|_{2}^{2}+\frac{1}{2}\left\|S-S^{*}\right\|_{2}^{2},
$$

where $S^{*}=\operatorname{col}\left(X^{*}, \Lambda^{*}, Z^{*}\right), X^{*}$ is the optimal solution to (8), $\Lambda^{*}$ is the optimal solution to (11), and $Z^{*}$ is the dual optimal solution to (12).

Notice that $X^{*}$ is a finite point from Assumption 2 Because the Slater's condition is satisfied with Assumption 2, the dual optimal solution $\lambda^{*}$ for (10) exists and is finite by Proposition 5.3.1 of Bertsekas (2009). Since the function $f_{i}\left(x_{i}\right)$ is strictly convex, $\nabla q_{i}\left(\lambda^{*}\right)=d_{i}-x_{i}^{*}$ by Theorem 2.87 of Ruszczynski (2006) and the saddle point property of (10). Then the KKT condition of (11) is $L \Lambda^{*}=\mathbf{0}$ and $D-X^{*}-L \Lambda^{*}-L Z^{*}=\mathbf{0}$. Hence, $L Z^{*}=D-X^{*}$ implies the finiteness of the dual optimal solution $Z^{*}$ for (11). Therefore, $S^{*}$ is a finite point.

In fact, with the KKT conditions to (10) and (11), $F\left(S^{*}\right)=$ $\operatorname{col}\left(\left(\nabla f\left(X^{*}\right)-\Lambda^{*}\right), \mathbf{0}_{n}, \mathbf{0}_{n}\right)$, and $-\nabla f\left(X^{*}\right)+\Lambda^{*} \in C_{\Omega}\left(X^{*}\right)$, we have

$$
H\left(S^{*}\right)=P_{\bar{\Omega}}\left(S^{*}-F\left(S^{*}\right)\right)=S^{*},-F\left(S^{*}\right) \in C_{\bar{\Omega}}\left(S^{*}\right) .
$$

Due to (2) and (3),

$$
\begin{aligned}
& -\langle F(S), H(S)-S\rangle-\frac{1}{2}\langle H(S)-S, H(S)-S\rangle \\
& =\frac{1}{2}\left[\|F(S)\|_{2}^{2}-\|F(s)+H(S)-S\|_{2}^{2}\right] \\
& =\frac{1}{2}\left[\|S-F(S)-S\|_{2}^{2}-\|H(S)-(S-F(S))\|_{2}^{2}\right] \\
& \geq \frac{1}{2}\|S-H(S)\|_{2}^{2} .
\end{aligned}
$$

Hence, $V_{g} \geq \frac{1}{2}\|S-H(S)\|_{2}^{2}+\frac{1}{2}\left\|S-S^{*}\right\| \geq 0$, and $V_{g}=0$ if and only if $S=S^{*}$.

By Theorem 3.2 of Fukushima (1992), any asymmetric variational inequality can be converted to a differentiable optimization problem. As a result,

$$
\dot{V}_{g}=\left(F(S)-\left[J_{F}(S)-I\right](H(S)-S)+S-S^{*}\right)^{T}(H(S)-S),
$$

where $J_{F}(S)$ is the Jacabian matrix of $F(S)$ defined as

$$
J_{F}(S)=\left(\begin{array}{ccc}
\nabla^{2} f(X) & -I & 0 \\
I & L & L \\
0 & -L & 0
\end{array}\right) .
$$

With Assumptions 1 and 3 ,

$S^{T} J_{F}(\bar{S}) S=X^{T} \nabla^{2} f(\bar{X}) X+\Lambda^{T} L \Lambda>0, \quad \forall \bar{S} \in \bar{\Omega}, \forall S \neq \mathbf{0} \in \mathbf{R}^{3 n}$.

With (2), taking $x=S-F(S)$ and $y=S^{*}$ gives $\langle S-F(S)-$ $\left.H(S), H(S)-S^{*}\right\rangle \geq 0$, which implies $\langle S-H(S)-F(S), H(S)-$ $\left.S+S-S^{*}\right\rangle \geq 0$. Hence, $-\|H(S)-S\|_{2}^{2}+\left\langle S-H(S), S-S^{*}\right\rangle+$ $\langle-F(S), H(S)-S\rangle+\left\langle-F(S), S-S^{*}\right\rangle \geq 0$, or equivalently,

$$
\left\langle S-H(S), S-S^{*}+F(S)\right\rangle \geq\|H(S)-S\|_{2}^{2}+\left\langle F(S), S-S^{*}\right\rangle .
$$

Consequently,

$$
\begin{aligned}
\dot{V}_{g} \leq & -(H(S)-S)^{T} J_{F}(S)(H(S)-S)+\|H(S)-S\|_{2}^{2} \\
& +\left\langle S-S^{*}+F(S), H(S)-S\right\rangle \\
\leq & -\left\langle F(S), S-S^{*}\right\rangle \\
\leq & -\left\langle F(S), S-S^{*}\right\rangle+\left\langle F\left(S^{*}\right), S-S^{*}\right\rangle-\left\langle F\left(S^{*}\right), S-S^{*}\right\rangle \\
\leq & -\left\langle F(S)-F\left(S^{*}\right), S-S^{*}\right\rangle-\left\langle F\left(S^{*}\right), S-S^{*}\right\rangle .
\end{aligned}
$$


In fact, $\left\langle F(S)-F\left(S^{\prime}\right), S-S^{\prime}\right\rangle=\left\langle\nabla f(X)-\nabla f\left(X^{\prime}\right), X-X^{\prime}\right\rangle+$ $\left\langle\Lambda-\Lambda^{\prime}, L\left(\Lambda-\Lambda^{\prime}\right)\right\rangle \geq 0, \forall S, S^{\prime} \in \bar{\Omega}$, because the local objective functions are convex, and the Laplacian matrix is positive semi-definite by Assumptions 1 and 3. With (16), we have $\left\langle F\left(S^{*}\right), S-S^{*}\right\rangle \geq 0$. Then $\dot{V}_{g} \leq 0$, and any finite equilibrium point $S^{*}$ of (9) is Lyapunov stable. Furthermore, there exists a forward compact invariance set given any finite initial points,

$$
I_{S}=\left\{\operatorname{col}(X, \Lambda, Z) \mid \frac{1}{2}\left\|S-S^{*}\right\| \leq V_{g}(S(0))\right\}
$$

Therefore, with the local Lipstchitz continuity of the objective functions' gradients in Assumption 1 and the nonexpansive property of projection operation (4), $P(S-F(S))-S$ is Lipstchitz over the compact set $I_{S}$ in (17). There exists a unique solution to (9) with time domain $[0, \infty)$. Also, the compactness and convexity of $I_{S}$ implies the existence of equilibrium point to dynamics (9) (referring to page 228 of Aubin \& Cellina (1984)). By Theorem 3.1 and without loss of generality, the equilibrium point is assumed to be $S^{*}$.

Furthermore, there exists $c^{*} \in C_{\Omega}\left(X^{*}\right)$ such that $-\nabla f\left(X^{*}\right)+$ $\Lambda^{*}=c^{*}, L Z^{*}=D-X^{*}$, and $\Lambda^{*}=\mathbf{1}_{n} \lambda^{*}$.

$$
\begin{aligned}
\dot{V}_{g} \leq & -\left\langle F(S), S-S^{*}\right\rangle=-\left\langle X-X^{*}, \nabla f(X)-\Lambda\right\rangle \\
& -\left\langle\Lambda-\Lambda^{*}, L \Lambda+L Z-\left(D-X^{*}\right)\right\rangle-\left\langle Z-Z^{*},-L \Lambda\right\rangle \\
\leq & -\left\langle X-X^{*}, \nabla f(X)-\Lambda-\nabla f\left(X^{*}\right)+\Lambda^{*}-c^{*}\right\rangle \\
& -\left\langle\Lambda-\Lambda^{*}, L\left(\Lambda-\Lambda^{*}\right)\right\rangle-\left\langle\Lambda-\Lambda^{*}, L\left(Z-Z^{*}\right)\right\rangle \\
& -\left\langle\Lambda-\Lambda^{*}, L Z^{*}-(D-X)\right\rangle-\left\langle Z-Z^{*},-L\left(\Lambda-\Lambda^{*}\right)\right\rangle \\
\leq & -\left\langle X-X^{*}, \nabla f(X)-\nabla f\left(X^{*}\right)\right\rangle+\left\langle X-X^{*}, c^{*}\right\rangle \\
& -\left\langle\Lambda-\Lambda^{*}, L\left(\Lambda-\Lambda^{*}\right)\right\rangle \\
\leq & -\left\langle X-X^{*}, \nabla f(X)-\nabla f\left(X^{*}\right)\right\rangle-\left\langle\Lambda-\Lambda^{*}, L\left(\Lambda-\Lambda^{*}\right)\right\rangle,
\end{aligned}
$$

where the last step follows from $\left\langle X-X^{*}, c^{*}\right\rangle \leq 0$. Denote the set of points satisfying $\dot{V}_{g}=0$ as $E_{g}=\left\{(X, \Lambda, Z) \mid \dot{V}_{g}=0\right\}$. Because the Hessian matrix of $\nabla^{2} f(X)$ is positive definite, $\nabla f(X)=\nabla f\left(X^{*}\right)+\int_{0}^{1} \nabla^{2} f\left(\tau X+(1-\tau) X^{*}\right)^{T}\left(X-X^{*}\right) d \tau$ and the null space for $L$ imply that

$$
E_{g}=\left\{(X, \Lambda, Z) \mid X=X^{*}, \Lambda \in \operatorname{span}\left\{\alpha \mathbf{1}_{n}\right\}\right\} .
$$

Then we claim that the maximal invariance set within the set $E_{g}$ is exactly the equilibrium point of (9). In fact, $\Lambda \in$ $\operatorname{span}\left\{\alpha \mathbf{1}_{n}\right\}$ implies $Z=Z^{*}$. Hence, $\dot{\Lambda}=L Z^{*}-\left(D-X^{*}\right)$. However, $L Z^{*}-\left(D-X^{*}\right)$ must be zero; otherwise $\Lambda$ will go to infinity, which contradicts that $E_{g}$ is a compact set within $I_{S}$. Thus, $\dot{\Lambda}=0$ and $\Lambda=\Lambda^{*}$.

By the LaSalle invariance principle and Lyapunov stability of the equilibrium point, the system (9) converges to its equilibrium point, which implies the conclusion.

Remark 3.5. Although an initialization-free algorithm has also been proposed and investigated for the DEDP, a special case of DRAO, in Cherukuri E Cortés (2014), algorithm (9) provides a different algorithm to address the DRAO problem without initialization. Additionally, algorithm (9) can handle general multi-dimensional LFCs explicitly with the projection operation, while Cherukuri E Cortés (2014) only addressed one-dimensional box constraints with a penalty method. Moreover, one agent was required to know the total
Table 1: Parameters setting of Example 3.6

\begin{tabular}{|l|l|l|l|}
\hline & $0 \sim 600 s$ & $600 s \sim 1200 s$ & $1200 s \sim$ \\
\hline$a_{1}, d_{1}$ & $(8,2),(8,2)$ & $(0.1,0.3),(8,2)$ & $(0.1,0.3),(12,-3)$ \\
\hline$a_{2}, d_{2}$ & $(4,7),(3,4)$ & $(-17,15),(3,4)$ & $(-17,15),(0,7)$ \\
\hline$a_{3}, d_{3}$ & $(0.13,8),(3,8)$ & $(0.13,8),(-5,12)$ & $(3,0.7),(-5,12)$ \\
\hline$a_{4}, d_{4}$ & $(4,20),(10,2)$ & $(4,20),(1,15)$ & $(5,17),(1,15)$ \\
\hline
\end{tabular}

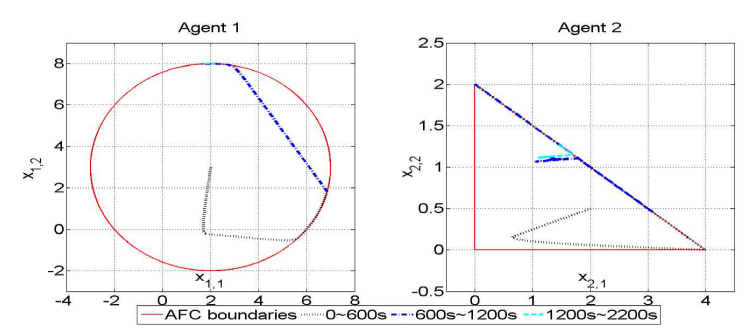

Figure 1: The trajectories of the allocations of agent 1 and agent 2.

network resource all the time in a time-varying resource case in Cherukuri E Cortés (2014), while each agent only knows its local resource in (9). Moreover, our techniques introduce a variational inequality viewpoint in addition to Lyapunov methods with the invariance principle.

The following example illustrates how (9) "adaptively" achieves the optimal resource allocation without reinitialization for a dynamical network. Notice that the following example cannot be directly addressed by the algorithm in Cherukuri \& Cortés (2014).

Example 3.6. Four agents cooperatively optimize problem (8). The allocation variable and resource data for agent $i$ are $x_{i}=$ $\left(x_{i, 1}, x_{i, 2}\right)^{T} \in \mathbf{R}^{2}, d_{i}=\left(d_{i, 1}, d_{i, 2}\right)^{T} \in \mathbf{R}^{2}$, respectively. The objective functions $f_{i}\left(x_{i}\right)$ are parameterized with $a_{i}=\left(a_{i, 1}, a_{i, 2}\right)^{T} \in$ $\mathbf{R}^{2}$ as follows:

$$
f_{i}\left(x_{i}\right)=\left(x_{i, 1}+a_{i, 1} x_{i, 2}\right)^{2}+x_{i, 1}+a_{i, 2} x_{i, 2}+0.001\left(x_{i, 1}^{2}+x_{i, 2}^{2}\right) .
$$

The LFCs of the four agents are given as follows: $\Omega_{1}=\left\{x_{1} \in\right.$ $\left.\mathbf{R}^{2} \mid\left(x_{1,1}-2\right)^{2}+\left(x_{1,2}-3\right)^{2} \leq 25\right\}, \Omega_{2}=\left\{x_{2} \in \mathbf{R}^{2} \mid x_{2,1} \geq 0, x_{2,1} \geq\right.$ $\left.0, x_{2,1}+2 x_{2,2} \leq 4\right\}, \Omega_{3}=\left\{x_{3} \in \mathbf{R}^{2} \mid 4 \leq x_{3,1} \leq 6,2 \leq x_{3,2} \leq 5\right\}$ and $\Omega_{4}=\left\{x_{4} \in \mathbf{R}^{2} \mid 0 \leq x_{4,1} \leq 15,0 \leq x_{4,2} \leq 20\right\}$, respectively, and their boundaries are shown in Figures 1 and 2

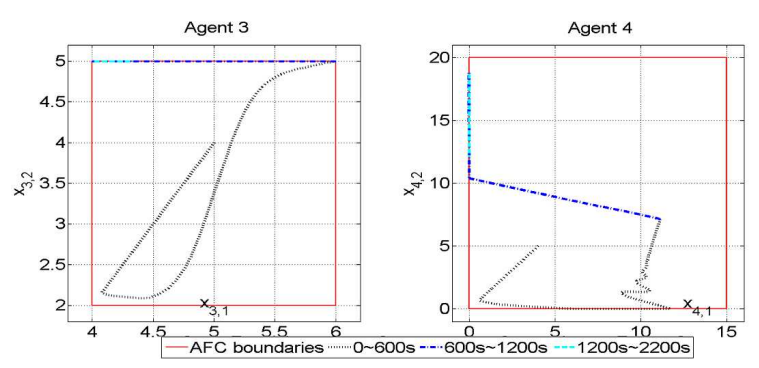

Figure 2: The trajectories of the allocations of agent 3 and agent 4. 

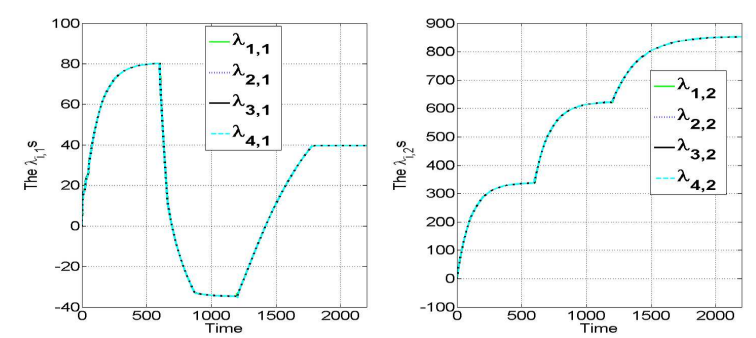

Figure 3: The trajectories of the Lagrangian multiplies $\lambda_{i}$ 's
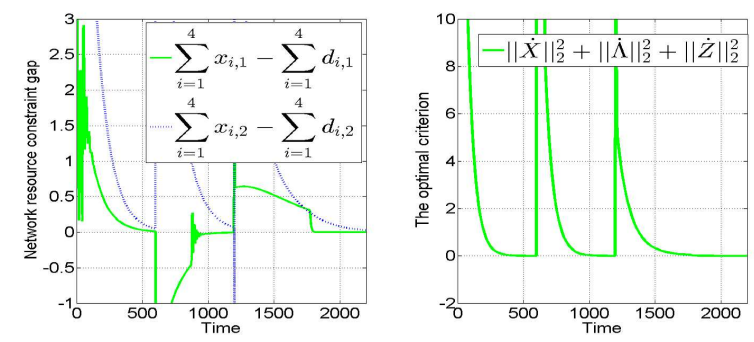

Figure 4: Network resource constraint and optimality condition

The agents share information with a ring graph $\mathcal{G}$ :

$$
1 \leftrightarrow 2 \leftrightarrow 3 \leftrightarrow 4 \leftrightarrow 1 .
$$

The initial allocation $x_{i}(0)$ of agent $i$ in algorithm (9) is randomly chosen within its $L F C$ set, and $\Lambda, Z$ are set with zero initial values. The data $a_{i}$ and $d_{i}$ switches as Table 3 while the allocation variables remain unchanged when the data switches. The simulation results are shown in Figures 12,3 and 4

Figures [1 and 2] show that the agents' allocation variables always remain within the corresponding $L F C$ sets, while Figure 3 shows that the Lagrangian multipliers reach consensus after the transient processes. Figure 4 demonstrates that the network resource constraint can be satisfied asymptotically even though it is violated each time the data/configuration changes, and Figure 4 also reveals that $\|\dot{X}\|_{2}^{2}+\|\dot{\Lambda}\|_{2}^{2}+\|\dot{Z}\|_{2}^{2}$ always converges to zero, guaranteeing the optimality of the resource allocations.

\section{Differentiated projected algorithm for DRAO}

In this section, the differentiated projection operator (6) is applied to derive an algorithm for (8). In fact, the projected dynamics based on the operator in (6) was firstly introduced in the study of constrained stochastic approximation in Dupuis \& Kushner (1987), and later was utilized to solve variational inequalities and constrained optimization problems in Nagurney \& Zhang (1995), Brogliato, Daniilidis. Lemarchal, \& Acary (2006), and Cherukuri, Mallada, \& Cortés (2016). Here the operator (6) is applied to the construction of the distributed resource allocation algorithm for agent $i$ given as follows:

\begin{tabular}{l}
$\overline{\text { Differentiated projected algorithm for agent } \mathbf{i}:}$ \\
\hline$\dot{x}_{i}=\prod_{\Omega_{i}}\left(x_{i},-\nabla f_{i}\left(x_{i}\right)+\lambda_{i}\right)$ \\
$\dot{\lambda}_{i}=-\sum_{j \in N_{i}}\left(\lambda_{i}-\lambda_{j}\right)-\sum_{j \in N_{i}}\left(z_{i}-z_{j}\right)+\left(d_{i}-x_{i}\right)$ \\
$\dot{z}_{i}=\sum_{j \in \mathcal{N}_{i}}\left(\lambda_{i}-\lambda_{j}\right)$ \\
\hline
\end{tabular}

Remark 4.1. The algorithm (18) is a direct extension of (9) by differentiating the projection operator, where each agent is required to project $-\nabla f_{i}\left(x_{i}\right)+\lambda_{i}$ onto the tangent cone $T_{\Omega_{i}}\left(x_{i}\right)$. Thereby, (18) has the additional burden for the tangent cone computation compared with (9). However, for some specific convex sets such as polyhedron, Euclidean ball, and boxes, it is not hard to get the close form of the tangent cone at any given point.

Similar to the algorithm (9), the algorithm (18) is also a distributed algorithm, and it does not need any initialization coordination procedure. Therefore, it can efficiently process online data for dynamical networks.

Although algorithm (18) is a discontinuous dynamical system, the solution to 18 is well-defined in the Caratheodory sense (an absolutely continuous function $\operatorname{col}(X(t), \Lambda(t), Z(t))$ : $[0, T] \rightarrow \mathbf{R}^{3 m n}$ is a solution of (18) if (18) is satisfied for almost all $t \in[0, T]$, referring to Definition 2.5 in Nagurney \& Zhang (1995)). The existence of an absolutely continuous solution to (18) can be found in Theorem 3.1 of Cojocaru \& Jonker (2004), and the condition when the solution can be extended to interval $[0, \infty]$ is given in Theorem 1 of Brogliato, Daniilidis, Lemarchal, \& Acary (2006).

The following result shows the correctness of algorithm (18).

Theorem 4.2. Suppose that Assumptions 13 hold. If the initial point $x_{i}(0) \in \Omega_{i}, \forall i \in \mathcal{N}$, then $x_{i}(t) \in \Omega_{i}, \forall t \geq 0, \forall i \in \mathcal{N}$, and there is the equilibrium point of the algorithm (18) with $X^{*}=\operatorname{col}\left(x_{1}^{*}, \ldots, x_{n}^{*}\right)$ as the optimal solution to (8).

Proof: Obviously, $\dot{x}_{i} \in T_{\Omega_{i}}\left(x_{i}\right), \quad \forall x_{i} \in \Omega_{i}$ according to Lemma 2.1 It follows that (18) has an absolutely continuous solution on interval $[0, \infty]$ by Theorem 1 of Brogliato, Daniilidis, Lemarchal, \& Acary (2006). Moreover, Theorem 3.2 of Cojocaru \& Jonker (2004) shows that the solution of (18) coincides with a slow solution of a differential inclusion. Given the initial point $x_{i}(0) \in \Omega_{i}, x_{i}(t) \in \Omega_{i}, \forall t \geq$ 0 holds in light of the viability theorem in Aubin \& Cellina (1984).

By Lemma 2.1, we have that $\Pi_{\Omega_{i}}\left(x_{i},-\nabla f_{i}\left(x_{i}\right)+\lambda_{i}\right)=0$ if at least one of the following cases is satisfied: (i): $x_{i} \in \operatorname{int}\left(\Omega_{i}\right)$, and $-\nabla f_{i}\left(x_{i}\right)+\lambda_{i}=0$; (ii): $x_{i} \in \partial \Omega_{i}$, and $-\nabla f_{i}\left(x_{i}\right)+\lambda_{i}=0$; (iii): $x_{i} \in \partial \Omega_{i}$, and $-\nabla f_{i}\left(x_{i}\right)+\lambda_{i} \in C_{\Omega_{i}}\left(x_{i}\right)$. Hence, $\Pi_{\Omega_{i}}\left(x_{i},-\nabla f_{i}\left(x_{i}\right)+\right.$ $\left.\lambda_{i}\right)=0$ implies $-\nabla f_{i}\left(x_{i}\right)+\lambda_{i} \in C_{\Omega_{i}}\left(x_{i}\right)$. Following similar analysis of Theorem 3.1, at the equilibrium point we have

$$
\begin{aligned}
& \Lambda^{*}=\mathbf{1}_{n} \otimes \lambda^{*}, \lambda^{*} \in \mathbf{R}^{m}, \quad x_{i}^{*} \in \Omega_{i} \\
& \left(L \otimes I_{m}\right) Z^{*}=D-X^{*}, \sum_{i \in \mathcal{N}} x_{i}^{*}=\sum_{i \in \mathcal{N}} d_{i}, \\
& -\nabla f_{i}\left(x_{i}^{*}\right)+\lambda^{*} \in C_{\Omega_{i}}\left(x_{i}^{*}\right) .
\end{aligned}
$$


Thus, the optimality condition (14) of (8) is satisfied by the equilibrium point of (18).

Next result shows the convergence of (18) when the local objective functions are strongly convex.

Theorem 4.3. Suppose that Assumptions 13 hold, and the local objective functions $f_{i}\left(x_{i}\right)$ are $\mu_{i}$-strongly convex functions with $k_{i}$-Lipschitz continuous gradients. Given bounded initial points $x_{i}(0) \in \Omega_{i}, \forall i \in \mathcal{N}$, the trajectories of algorithm (18) converge to its equilibrium point. Furthermore, if there are no LFCs (that is, $\Omega_{i}=\mathbf{R}^{m}, i=1, \cdots, n$ ), then algorithm (18) exponentially converges to its equilibrium point.

Proof: We still take $m=1$ without loss of generality. At first, we show the convergence of (18). By Lemma 2.1,

$$
\Pi_{\Omega_{i}}\left(x_{i},-\nabla f_{i}\left(x_{i}\right)+\lambda_{i}\right)=-\nabla f_{i}\left(x_{i}\right)+\lambda_{i}-\beta\left(x_{i}\right) n_{i}\left(x_{i}\right),
$$

where $n_{i}\left(x_{i}\right) \in c_{\Omega_{i}}\left(x_{i}\right), \beta\left(x_{i}\right) \geq 0$. Notice that there exist $\beta\left(x_{i}^{*}\right) \geq 0$ and $n_{i}\left(x_{i}^{*}\right) \in c_{\Omega_{i}}\left(x_{i}^{*}\right)$ at the equilibrium point such that $\nabla f_{i}\left(x_{i}^{*}\right)-\lambda^{*}=-\beta\left(x_{i}^{*}\right) n_{i}\left(x_{i}^{*}\right)$.

Define the following variables

$$
\begin{array}{lll}
Y=X-X^{*}, & \theta=[r R]^{T} Y, & Y=[r R] \theta, \\
V=\Lambda-\Lambda^{*}, & \eta=[r R]^{T} V, & V=[r R] \eta, \\
W=Z-Z^{*}, & \delta=[r R]^{T} W, & W=[r R] \delta,
\end{array}
$$

with $r=\frac{1}{\sqrt{n}} \mathbf{1}_{n}$ and $r^{T} R=\mathbf{0}_{n}^{T}$ such that $R^{T} R=I_{n-1}$ and $R R^{T}=$ $I_{n}-\frac{1}{n} \mathbf{1}_{n} \mathbf{1}_{n}^{T}$. We partition the variables $\theta, \eta, \delta$ as $\operatorname{col}\left(\theta_{1}, \theta_{2}\right)$, $\operatorname{col}\left(\eta_{1}, \eta_{2}\right), \operatorname{col}\left(\delta_{1}, \delta_{2}\right)$ with $\theta_{1}, \eta_{1}, \delta_{1} \in \mathbf{R}$ and $\theta_{2}, \eta_{2}, \delta_{2} \in \mathbf{R}^{n-1}$.

Then the dynamics of the variables $\theta, \eta, \delta$ can be derived with (18), (19), 20) and (21) as follows,

$$
\begin{array}{ll}
\dot{\theta}_{1}=-r^{T} h+\eta_{1} ; & \dot{\theta}_{2}=-R^{T} h+\eta_{2} ; \\
\dot{\eta}_{1}=-\theta_{1} ; & \dot{\eta}_{2}=-\theta_{2}-R^{T} L R \eta_{2}-R^{T} L R \delta_{2} ; \\
\dot{\delta}_{1}=0 ; & \dot{\delta}_{2}=R^{T} L R \eta_{2},
\end{array}
$$

where $h=\nabla f\left(Y+X^{*}\right)-\nabla f\left(X^{*}\right)+N_{\Omega}(X)-N_{\Omega}\left(X^{*}\right)$, $N_{\Omega}(X)=\operatorname{col}\left(\beta\left(x_{1}\right) n_{1}\left(x_{1}\right), \ldots, \beta\left(x_{n}\right) n_{n}\left(x_{n}\right)\right)$, and $N_{\Omega}\left(X^{*}\right)=$ $\operatorname{col}\left(\beta\left(x_{1}^{*}\right) n_{1}\left(x_{1}^{*}\right), \ldots, \beta\left(x_{n}^{*}\right) n_{n}\left(x_{n}^{*}\right)\right)$.

Construct the following function

$$
\begin{aligned}
V_{1}^{s} & =\frac{1}{2} \alpha\left(\theta^{T} \theta+\eta^{T} \eta\right)+\frac{1}{2}(\alpha+\gamma) \delta_{2}^{T} \delta_{2} \\
& +\frac{1}{2} \gamma\left(\eta_{2}+\delta_{2}\right)^{T}\left(\eta_{2}+\delta_{2}\right),
\end{aligned}
$$

where $\alpha, \gamma$ are positive constants to be determined later. Obviously, $\frac{1}{2} \alpha\|p\|_{2}^{2} \leq V_{1}^{s} \leq\left(\frac{1}{2}(\alpha+\gamma)+\gamma\right)\|p\|_{2}^{2}$ where $p=\operatorname{col}\left(\theta, \eta, \delta_{2}\right)$. The derivative of $V_{1}^{s}$ along (18) is

$$
\begin{aligned}
\dot{V}_{1}^{s} & =\alpha\left(-Y^{T} h-\eta_{2} R^{T} L R \eta_{2}-\eta_{2} R^{T} L R \delta_{2}\right) \\
& +\gamma\left(-\eta_{2}^{T} \theta_{2}-\eta_{2} R^{T} L R \delta_{2}-\delta_{2} \theta_{2}-\delta_{2}^{T} R^{T} L R \delta_{2}\right) \\
& +(\alpha+\gamma) \delta_{2}^{T} R^{T} L R \eta_{2}
\end{aligned}
$$

Because $n_{i}\left(x_{i}^{*}\right) \in c_{\Omega_{i}}\left(x_{i}^{*}\right)$ and $\beta\left(x_{i}^{*}\right) \geq 0, \beta\left(x_{i}^{*}\right)\left\langle x_{i}-\right.$ $\left.x_{i}^{*}, n_{i}\left(x_{i}^{*}\right)\right\rangle \leq 0$. Moreover, $n_{i}\left(x_{i}\right) \in c_{\Omega_{i}}\left(x_{i}\right)$ and $\beta\left(x_{i}\right) \geq 0$ imply that $\beta\left(x_{i}\right)\left\langle x_{i}-x_{i}^{*}, n_{i}\left(x_{i}\right)\right\rangle \geq 0$. Because the local objective functions are strongly convex, $Y^{T} h=\left(X-X^{*}\right)^{T}(\nabla f(X)-$ $\left.\nabla f\left(X^{*}\right)+N_{\Omega}(X)-N_{\Omega}\left(X^{*}\right)\right)=\left(X-X^{*}\right)^{T}\left(\nabla f(X)-\nabla f\left(X^{*}\right)\right)+$ $\sum_{i=1}^{n}\left\langle x_{i}-x_{i}^{*},+\beta\left(x_{i}\right) n_{i}\left(x_{i}\right)\right\rangle+\sum_{i=1}^{n}\left\langle x_{i}-x_{i}^{*},-\beta\left(x_{i}^{*}\right) n_{i}\left(x_{i}^{*}\right)\right\rangle \geq \bar{\mu} \theta^{T} \theta$ where $\bar{\mu}=\min \left\{\mu_{1}, \cdots, \mu_{n}\right\}$.
Denote $s_{1} \leq s_{2} \leq \ldots \leq s_{n}$ as the ordered eigenvalues of Laplacian matrix $L$. Obviously, $s_{1}=0$ and $s_{2}>0$ when the graph $\mathcal{G}$ is connected. By (7),

$$
\dot{V}_{1}^{s} \leq-\alpha \bar{\mu} \theta^{T} \theta-\alpha s_{2} \eta_{2}^{T} \eta_{2}-\gamma s_{2} \delta_{2}^{T} \delta_{2}-\gamma \eta_{2}^{T} \theta_{2}-\gamma \delta_{2} \theta_{2}
$$

According to $-\gamma \eta_{2}^{T} \theta_{2} \leq \frac{1}{2} \gamma^{2} \theta_{2}^{T} \theta_{2}+\frac{1}{2} \eta_{2}^{T} \eta_{2}$, and $-\gamma \delta_{2}^{T} \theta_{2} \leq$ $\frac{1}{2} \gamma^{2} \theta_{2}^{T} \theta_{2}+\frac{1}{2} \delta_{2}^{T} \delta_{2}$, we have

$$
\dot{V}_{1}^{s} \leq-\left(\alpha \bar{\mu}-\gamma^{2}\right) \theta^{T} \theta-\left(\alpha s_{2}-\frac{1}{2}\right) \eta_{2}^{T} \eta_{2}-\left(\gamma s_{2}-\frac{1}{2}\right) \delta_{2}^{T} \delta_{2} .
$$

Take $\gamma$ and $\alpha$ such that $\gamma>\frac{1}{2 s_{2}}$, and $\alpha>\max \left\{\frac{\gamma^{2}}{\bar{\mu}}, \frac{1}{2 s_{2}}\right\}$. Then we have $\dot{V}_{1}^{s}<0$, which leads to the convergence of algorithm (18).

Next, we estimate the convergence rate of (18) when $\Omega_{i}=$ $\mathbf{R}^{m}, i=1, \cdots, n$. In this case $\Pi_{\Omega_{i}}\left(x_{i},-\nabla f_{i}\left(x_{i}\right)+\lambda_{i}\right)=-\nabla f_{i}\left(x_{i}\right)+$ $\lambda_{i}$, and $\beta\left(x_{i}\right) n_{i}\left(x_{i}\right)=\mathbf{0}$. Still take $V_{1}^{s}$ in (23), and then (24) still holds in this case.

Take $V_{2}^{s}=\varepsilon(\theta-\eta)^{T}(\theta-\eta)$, and we have

$$
\begin{aligned}
\dot{V}_{2}^{s}= & -\varepsilon Y^{T} h+\varepsilon \theta^{T} \theta+\varepsilon \theta_{2} R^{T} L R \eta_{2}+\varepsilon \theta_{2} R^{T} L R \delta_{2} \\
& +\varepsilon \eta^{T}[r, R]^{T} h-\varepsilon \eta^{T} \eta-\varepsilon \eta_{2} R^{T} L R \eta_{2}-\varepsilon \eta_{2} R^{T} L R \delta_{2} \\
\leq & -(\varepsilon \bar{\mu}-\varepsilon) \theta^{T} \theta+\frac{1}{2} \varepsilon s_{n}^{2} \theta^{T} \theta+\frac{1}{2} \varepsilon \eta_{2}^{T} \eta_{2}^{T}+\frac{1}{2} \varepsilon s_{n}^{2} \theta^{T} \theta \\
& +\frac{1}{2} \varepsilon \delta_{2} \delta_{2}^{T}+\frac{1}{2} \varepsilon \eta^{T} \eta+\frac{1}{2} \varepsilon M^{2} \theta^{T} \theta-\varepsilon \eta^{T} \eta-\varepsilon s_{2} \eta_{2}^{T} \eta_{2} \\
& +\frac{1}{2} \varepsilon s_{n}^{2} \eta_{2}^{T} \eta_{2}+\frac{1}{2} \varepsilon \delta_{2}^{T} \delta_{2} \\
\leq & -\varepsilon\left(\bar{\mu}-1-s_{n}^{2}-\frac{1}{2} M^{2}\right) \theta^{T} \theta-\frac{1}{2} \varepsilon \eta^{T} \eta \\
& +\varepsilon \delta_{2}^{T} \delta_{2}-\varepsilon\left(s_{2}-\frac{1}{2}-\frac{1}{2} s_{n}^{2}\right) \eta_{2}^{T} \eta_{2},
\end{aligned}
$$

with $M=\max \left\{k_{1}, \cdots, k_{n}\right\}$, by using the inequality $x^{T} y \leq$ $\frac{1}{2}\|x\|_{2}^{2}+\|y\|_{2}^{2}$ and (7) in the first step of (25).

With $V^{s}=V_{1}^{s}+V_{2}^{s}$, it is easy to see that

$$
\begin{aligned}
\dot{V}^{s} & =-\left(\alpha \bar{\mu}-\gamma^{2}+\varepsilon\left(\bar{\mu}-1-s_{n}^{2}-\frac{1}{2} M^{2}\right)\right) \theta^{T} \theta \\
& -\frac{1}{2} \varepsilon \eta^{T} \eta-\left(\gamma s_{2}-\frac{1}{2}-\varepsilon\right) \delta_{2}^{T} \delta_{2} \\
& -\left(\alpha s_{2}-\frac{1}{2}+\varepsilon\left(s_{2}-\frac{1}{2}-\frac{1}{2} s_{n}^{2}\right)\right) \eta_{2}^{T} \eta_{2} .
\end{aligned}
$$

Choose $\gamma \geq \frac{3 \varepsilon+1}{2 s_{2}}$ such that $\gamma_{s_{2}}-\frac{1}{2}-\varepsilon \geq \frac{1}{2} \varepsilon$. Select $\alpha$ such that

$$
\alpha \bar{\mu}-\gamma^{2}+\varepsilon\left(\bar{\mu}-1-s_{n}^{2}-\frac{1}{2} M^{2}\right) \geq \frac{1}{2} \varepsilon,
$$

and

$$
\alpha s_{2}-\frac{1}{2}+\varepsilon\left(s_{2}-\frac{1}{2}-\frac{1}{2} s_{n}^{2}\right) \geq 0
$$

As a result,

$$
\dot{V}^{s} \leq \frac{1}{2} \varepsilon\|p\|_{2}^{2} .
$$

Then, with $\frac{1}{2} \alpha\|p\|_{2}^{2} \leq V^{s} \leq\left(\frac{1}{2}(\alpha+\gamma)+\gamma+2 \varepsilon\right)\|p\|_{2}^{2}$, we have

$$
\|p\| \leq \sqrt{\frac{\alpha+3 \gamma+4 \varepsilon}{\alpha}}\|p(0)\| e^{-\frac{2 \varepsilon}{\alpha+3 \gamma+4 \varepsilon} t},
$$

which leads to the exponential convergence of algorithm (18) to its equilibrium point.

Remark 4.4. In fact, the exponential convergence speed $\frac{2 \varepsilon}{(\alpha+3 \gamma+4 \varepsilon)}$ in $(28)$ can be estimated by solving the following optimization problem

$$
\max _{\alpha, \gamma, \varepsilon \geq 0} \frac{2 \varepsilon}{(\alpha+3 \gamma+4 \varepsilon)} \quad \text { s. } t . \gamma \geq \frac{3 \varepsilon+1}{2 s_{2}}, \text { (26), (27). }
$$


To get a simple estimation, we take $\gamma=\frac{3 \varepsilon+1}{2 s_{2}}$. Denote $\varrho_{1}=\left(s_{n}^{2}+1-2 s_{2}\right)$ and $\varrho_{2}=s_{2}^{2}\left(6+4 s_{n}^{2}+2 M^{2}-4 \bar{\mu}\right)$. With taking $\alpha=\max \left\{\frac{1+\varepsilon \varrho_{1}}{2 s_{2}}, \frac{9 \varepsilon^{2}+\varepsilon\left(6+\varrho_{2}\right)+1}{4 s_{2}^{2} \bar{\mu}}\right\}$, we have $\frac{2 \varepsilon}{(\alpha+3 \gamma+4 \varepsilon)} \geq$ $\min \left\{\frac{2 s_{2}}{8+6 s_{2}+s_{n}^{2}}, \frac{4 s_{2}^{2} \bar{\mu}}{\left(3+2 s_{n}^{2}+M^{2}+6 \bar{\mu}\right) s_{2}^{2}+9 \bar{\mu} s_{2}+3 \sqrt{6 \bar{\mu} s_{2}+1}+3}\right\}$.

\section{Distributed economic dispatch in power grids}

In this section, the algorithm proposed in Section 4 is applied to the DEDP in power grids to find the optimal secure generation allocations for power balancing in a distributed manner. Example 5.2 is given to show that the distributed algorithm can efficiently adapt to online network data/configuration changes, including load demands, generation costs/capacities, and plugging-in/leaving-off of buses, while Example 5.3 with a large-scale network illustrates the scalability of the proposed algorithm.

Suppose that there exist control areas $\mathcal{N}=\{1, \ldots, n\}$ with area $i$ having local generators to supply power $P_{i}^{g} \in \mathbf{R}_{\geq 0}$ and local load demands $P_{i}^{d} \in \mathbf{R}_{\geq 0}$ to be met. The local generation must be kept within the capacity or security bounds $\underline{P}_{i} \leq P_{i}^{g} \leq \bar{P}_{i}, \underline{P}_{i}, \bar{P}_{i} \in \mathbf{R}_{\geq 0} . f_{i}\left(P_{i}^{g}\right): \mathbf{R}_{\geq 0} \rightarrow \mathbf{R}$ represents the local generation cost in control area $i$ with respect to its local generation $P_{i}^{g}$, and it satisfies Assumption 1 Then the DEDP formulation can be derived in the form of 8

\begin{tabular}{l}
\hline Distributed Economic Dispatch Problem : \\
$\min _{P_{i}^{g}, i \in \mathcal{N}} f\left(P^{g}\right)=\sum_{i \in \mathcal{N}} f_{i}\left(P_{i}^{g}\right)$ \\
subject to $\sum_{i \in \mathcal{N}} P_{i}^{g}=\sum_{i \in \mathcal{N}} P_{i}^{d}$ \\
$\underline{P}_{i} \leq P_{i}^{g} \leq \bar{P}_{i}, \quad \mathcal{N}=\{1, \ldots, n\}$ \\
\hline
\end{tabular}

Here a multi-agent network is introduced to solve the DEDP (29) motivated by recent DEDP works like Cherukuri, \& Cortés (2015), Cherukuri \& Cortés (2014), Zhao, Topcu, Li, \& Low (2014), Cavraro, Carli, \& Zampieri (2014) and Zhang, Liu, Wang, Liu, \& Ferrese (2015). Agent $i$ is responsible to decide the generation $P_{i}^{g}$ in control area $i$ to minimize the global cost as the sum of all individuals' generation costs, while meeting the total load demands within its capacity bounds. In addition, each agent can react to the changing local environment in real time, and adapt its own behavior or preference by adjusting its local data, including $P_{i}^{d}, \underline{P}_{i}, \bar{P}_{i}, f_{i}\left(P_{i}^{g}\right)$. The agents can also share information with their neighbors to facilitate the cooperations.

Then applying (18) to 29), the distributed algorithm for agent $i$ is

$$
\begin{aligned}
& \hline \text { Distributed algorithm for agent } \mathbf{i}: \\
& \hline \dot{P}_{i}^{g}=\left[-\nabla f_{i}\left(P_{i}^{g}\right)+\lambda_{i}\right]_{P_{i}^{g}-P_{i}^{g}}^{\bar{P}_{i}} \\
& \dot{\lambda}_{i}=-\sum_{j \in \mathcal{N}_{i}}\left(\lambda_{i}-\lambda_{j}\right)-\sum_{j \in \mathcal{N}_{i}}\left(z_{i}-z_{j}\right)+\left(P_{i}^{d}-P_{i}^{g}\right) \\
& \dot{z}_{i}=\sum_{j \in \mathcal{N}_{i}}\left(\lambda_{i}-\lambda_{j}\right)
\end{aligned}
$$

where $[v]_{x-c_{1}}^{c_{2}-x}=0$ if $x-c_{1}=0$ and $v \leq 0$ or $c_{2}-x=0$ and $v \geq 0$, otherwise $[v]_{x-c_{1}}^{c_{2}-x}=v$.
The algorithm (30) ensures generation capacity bounds explicitly, and converges without the initialization procedure, which is crucially important for the "plug-and-play" operation in the future smart grid.

Remark 5.1. The optimization problem (8) can also be applied to model the multi-period demand response in power systems. The objective functions describe the dis-utility of cutting loads in each area. The resource constraint specifies the amount of loads to be shed in the multi-periods. Particularly, the local load shedding constraints concern with the total power demands in the multi-periods and other specifications of that area. Then, the previous algorithms (9) and (18) can be applied to solve this multi-dimensional DRAO with general LFCs in a distributed manner. This issue is interesting but beyond the scope of this paper, and will be discussed elsewhere.

The following two examples are presented to further show the online adaptation property and scalability of our algorithm. Firstly, the standard IEEE 118-bus system is adopted to illustrate the performance of algorithm (30).

Example 5.2 (Optimality and adaptability). Consider the DEDP (29) in the IEEE 118-bus system with 54 generators. Each generator has a local quadratic generation cost function as $f_{i}\left(P_{i}^{g}\right)=a_{i} P_{i}^{g 2}+b_{i} P_{i}^{g}+c_{i}$, whose coefficients belong to the intervals $a_{i} \in[0.0024,0.0679]\left(M \$ / M W^{2}\right)$, $b_{i} \in[8.3391,37.6968](M \$ / M W)$, and $c_{i} \in[6.78,74.33](M \$)$. The generation capacity bounds of the generators are drawn from $\underline{P}_{i} \in[5,150](M W)$ and $\bar{P}_{i} \in[150,400](M W)$, while the load of each bus varies as $P_{i}^{d} \in[0,300](M W)$.

The corresponding agents share information on an undirected ring graph with additional undirected edges $(1,4)$, $(15,25),(25,35),(35,45)$ and $(45,50)$. The simulations are performed with the differentiated projected algorithm (30). The initial generation $P_{i}^{g}$ in algorithm (30) is set within its local capacity bounds, while variables $\lambda_{i}$ 's and $z_{i}$ 's are set with zero initial values.

Next explains how the network data/configuration changes at different times.

- Load variations: At time 100s, 18 buses are randomly chosen with randomly varying their loads by $-20 \sim$ $+20 \%$.

- Generation capacity variations: At time 200s, 18 generators randomly vary their capacity lower bounds by $-50 \sim+50 \%$, and another 18 generators randomly vary their capacity upper bounds by $-20 \sim+20 \%$.

- Generation cost variations: At time 300 s, 18 generators randomly vary their $a_{i}$ by $0 \sim 50 \%$, and another 18 generators randomly vary their $b_{i}$ by $-50 \sim 0 \%$.

- Leaving-off of buses: At time 400s, generator 2 and 3 disconnect from the system, and the communication edges associated to them are also removed. 

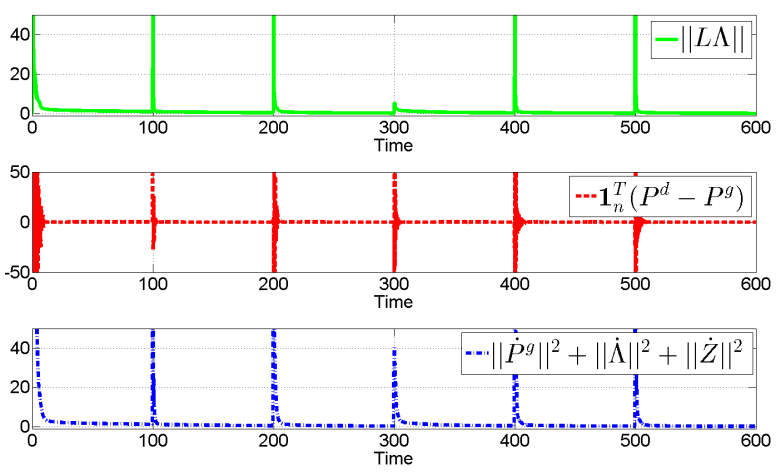

Figure 5: Algorithm performance indexes: (i), $\|L \Lambda\|$ 's always decrease to zero even with different $L$ 's, implying that $\lambda^{\prime} s$ always reach consensus. (ii), The power balance constraint (i.e., resource constraint) is violated if any network configuration changes. But the power balance gap $\mathbf{1}_{n}^{T}\left(P^{d}-P^{g}\right)$ asymptotically decreases to zero, even without any re-initialization coordination. (iii), The optimality condition $\left\|\dot{P}^{g}\right\|^{2}+\|\dot{\Lambda}\|^{2}+\|\dot{Z}\|^{2}=0$ can always be satisfied asymptotically, implying the economic efficiency of the generation dispatch.

- Plugging-in of bus: At time 500s, generator 3 plugs in the system with re-generated configurations $a_{i}, b_{i}, c_{i}, P_{i}^{d}, \underline{P}_{i}$, and $\bar{P}_{i}$. The undirected edge $(3,4)$ is also added to the communication graph.

When the data/configuration changes, each agent only projects its local generation onto to its local capacity bounds if necessary. The trajectrories of dynamics (30) are derived with a first-order Euler discretization using Matlab. The trajectories of some algorithm performance indexes are shown in Figure 5 It indicates that algorithm (30) can adaptively find the optimal solutions to 29] in a fully distributed way, even without any initialization coordination procedure, when the network data or configuration changes.

Example 5.3 (Scalability). This example considers a network of 1000 control areas to achieve economic dispatch during a normal day. Control area $i$ has cost function $f_{i}\left(P_{i}^{g}\right)=a_{i} P_{i}^{g 2}+b_{i} P_{i}^{g}$ as well as generation capacity upper bound $\bar{P}_{i}$ and lower bound $\underline{P}_{i}$. The control areas are divided equally into two groups. The first group, named as fuel group, is mainly supported with traditional thermal generators, and has relatively higher generation costs and larger capacity ranges with the nominal values of $a_{i}, b_{i}, \underline{P}_{i}, \bar{P}_{i}$ randomly drawn from the intervals, $[3,7]\left(M \$ / M W^{2}\right)$, $[5,9](M \$ / M W),[2,6](M W),[15,23](M W)$, respectively. The second group, named as renewable group, is mainly supported with renewable energies, and has lower generation costs and smaller capacity ranges with the nominal values of $a_{i}, b_{i}, \underline{P}_{i}, \bar{P}_{i}$ randomly drawn from the intervals, $\left[\frac{1}{2}, 2\right]\left(M \$ / M W^{2}\right)$, $\left[\frac{1}{2}, 4\right](M \$ / M W), \quad[0,1](M W), \quad\left[\frac{3}{2}, 7\right](M W)$, respectively.

The daily 96-point load data (15 minutes for each period) of each control area is generated from a typical load curve for a distribution system added with certain random perturbations. The total load curve of the network is given in Fig 6 In each period, $10 \%$ of the control areas in the renewable group are randomly chosen to change their generation costs and capacities like Example 5.2 with the variations less that $\pm 20 \%$ of its

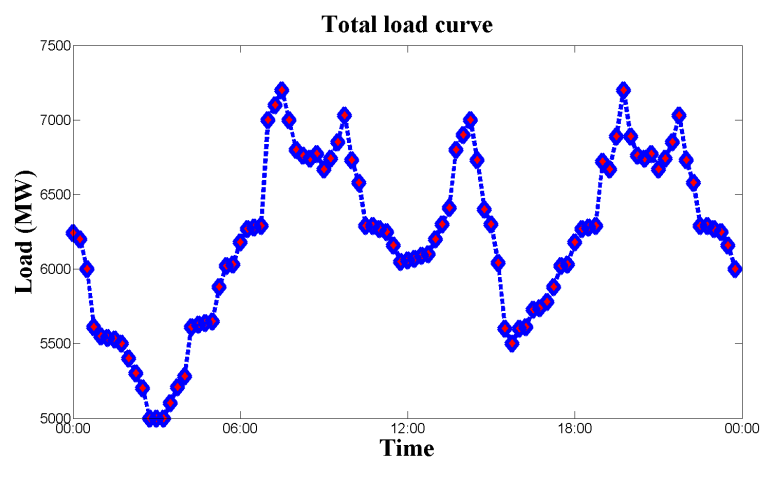

Figure 6: The total load curve
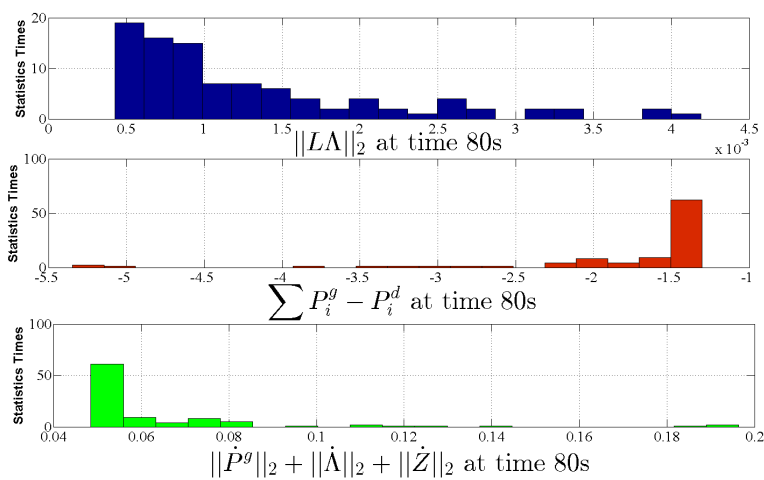

Figure 7: The performance indexes at time $t=80 \mathrm{~s}$ in histograms: (i), The consensus error $\|L \Lambda\|_{2}$ at $t=80 \mathrm{~s}$ always decreases to a rather lower level due to the second-order proportional-integral consensus dynamics. (ii), The power balance is almost achieved at $t=80 \mathrm{~s}$ without the initialization coordination. (iii), The optimality condition can always be satisfied at $t=80 \mathrm{~s}$.

nominal values.

In each period, a connected graph is re-generated with random graph model $\mathcal{G}(1000, \mathbb{P})$ as the information sharing graph of that period. In $\mathcal{G}(1000, \mathbb{P})$, every possible edge occurs independently with the probability of $\mathbb{P}$. Here, the probability $\mathbb{P}$ in each period is randomly drawn from the interval [0.0015, 0.005].

For each period, the computation time is set as 80 s. Figure 7 shows the histogram of consensus error $\|L \Lambda\|_{2}$, power balance gap $\sum P_{i}^{g}-\sum P_{i}^{d}$ and optimality condition $\left\|\dot{P}^{g}\right\|_{2}+\|\dot{\Lambda}\|_{2}+\|\dot{Z}\|_{2}$ at time $80 \mathrm{~s}$. It indicates that the agents can always find the economic power dispatch with varying loads and generation costs/capacities, and evidently demonstrates the scalability of the proposed method.

\section{Conclusions}

In this paper, a class of projected continuous-time distributed algorithms have been proposed to solve resource allocation optimization problems with the consideration of LFCs. The proposed algorithms are scalable and free of initialization coordination procedure, and therefore, are adaptable to working condition variations. These salient features have important implications in the DEDP in power systems. Firstly it allows quite 
general non-box LFCs, which is crucial when inverter-based devices are involved because their LFCs are usually depicted in a quadratic form. Secondly, our method is initialization free, which may facilitate the implementation of the so-called "plugand-play" operation for future smart grids in a dynamic environment. Our application examples illustrate such implications, showing an appealing potential in the smart operation of future power grids.

We would like to note that many challenging DRAO problems still remain to be investigated, including the design of algorithms for the non-smooth objective functions based on differential inclusions, the estimation of convergence rates for the proposed algorithms with general LFCs, and the development of stochastic algorithms to achieve the DRAO with noisy data observations. Furthermore, inspired by Zhao, Topcu, Li, \& Low (2014), we also hope to combine our algorithms with physical dynamics of power grids to derive a more comprehensive solution for the DEDP in power systems.

\section{References}

\section{References}

Arrow, K., Huwicz, L., \& Uzawa, H. (1958). Studies in Linear and Non-linear Programming, Stanford University Press.

Arrow, K. J., \& Hurwicz, L. (1960). Decentralization and Computation in Resource Allocation. Economics and Econometrics, 34-104, University of North Carolina Press.

Aubin, J.P., \& Cellina, A. (1984). Differential Inclusions, Berlin: SpringerVerlag.

Beck, A., Nedic, A., Ozdaglar, A., \& Teboulle, M. (2014). Optimal Distributed Gradient Methods for Network Resource Allocation Problems. IEEE Transactions on Control of Network Systems, 1(1),64-74.

Bertsekas, D. P. (2009). Convex Optimization Theory, MIT: Athena Scientific.

Bhaya, A., \& Kaszkurewicz, E. (2006). Control perspectives on numerical algorithms and matrix problems, 10, SIAM.

Brogliato, B., Daniilidis, A., Lemarchal, C., \& Acary, V. (2006). On the equivalence between complementarity systems, projected systems and differential inclusions. Systems $\mathcal{E}$ Control Letters, 55(1), 45-51.

Cavraro, G., Carli, R., \& Zampieri, S. (2014). A distributed control algorithm for the minimization of the power generation cost in smart micro-grid. IEEE 53rd Annual Conference on Decision and Control (CDC), Los Angeles, USA (pp. 5642-5647).

Cherukuri, A., \& Cortés, J. (2014) Initialization-free distributed coordination for economic dispatch under varying loads and generator commitment. Available in arXiv at http://arxiv.org/abs/1409.4382

Cherukuri, A., \& Cortés, J. (2015). Distributed generator coordination for initialization and anytime optimization in economic dispatch. IEEE Transactions on Control of Network Systems, 2(3): 226 - 237.

Cherukuri, A, Mallada E, \& Cortés J. (2016). Asymptotic convergence of constrained primal-dual dynamics. Systems $\mathcal{E}$ Control Letters, 87: 10-15.

Cojocaru, M. G., \& Jonker, L. (2004). Existence of solutions to projected differential equations in Hilbert spaces. Proceedings of the American Mathematical Society, 132(1), 183-193.

D'Amico, A., Sanguinetti, L. \& Palomar, D.P., (2014). Convex separable problems with linear constraints in signal processing and communictions, IEEE transaction on signal processing, 62(22), 6045-6058.

Droge, G., Kawashima, H., \& Egerstedt, M. B. (2014). Continuous-time proportional-integral distributed optimisation for networked systems. Journal of Control and Decision, 1(3), 191-213.

Dupuis, P., \& Kushner, H. J. (1987). Asymptotic behavior of constrained stochastic approximations via the theory of large deviations. Probability theory and related fields, 75(2), 223-244.

Feijer, D., \& Paganini, F. (2010). Stability of primalCdual gradient dynamics and applications to network optimization. Automatica, 46(12), 1974-1981.
Ferragut, A., \& Paganini, F. (2014). Network resource allocation for users with multiple connections: fairness and stability. IEEE/ACM Transactions on Networking (TON), 22(2), 349-362.

Fukushima, M. (1992). Equivalent differentiable optimization problems and descent methods for asymmetric variational inequality problems, Mathematical programming, 53(1-3), 99-110.

Gao, X. B. (2003). Exponential stabiliby of globally projected dynamic systems. IEEE Transactions on Neural Networks, 14(2), 426-431.

Ghadimi, E., Shames, I., \& Johansson, M. (2013). Multi-step gradient methods for networked optimization. IEEE Transactions on Signal Processing, 61(21), 5417-5429.

Gharesifard, B., \& Cortés, J. (2014). Distributed continuous-time convex optimization on weight-balanced digraphs. IEEE Transactions on Automatic Control, 59(3), 781-786.

Heal, G. M. (1969). Planning without Prices. Review of Economic Studies, 36(107), 347-362.

Ho, Y. C., Servi, L., \& Suri, R. (1980). A class of center-free resource allocation algorithms. Large Scale Systems, 1: 51-62.

Ibaraki, T., \& Katoh, N. (1988). Resource Allocation Problems: Algorithmic Approaches, Cambridge: MIT Press.

Johari, R., \& Tsitsiklis, J. N. (2004). Efficiency Loss in a Network Resource Allocation Game, Mathematics of operations research, 29(3), 407C435.

Kia, S., Cortés, J., \& Martinez, S. (2015). Distributed convex optimization via continuous-time coordination algorithms with discrete-time communication. Automatica, 55, 254-264.

Lakshmanan, H., \& Farias, D. P. (2008). Decentralized Resource Allocation in Dynamic Networks of Agents. SIAM Journal of Optimization, 19(2), 911940.

Liao, L. Z., Qi, H., \& Qi, L. (2004). Neurodynamical optimization. Journal of Global Optimization, 28(2), 175-195.

Liu, Q., \& Wang, J. (2015). A Second-order Multi-agent Network for BoundConstrained Distributed Optimization. IEEE Transactions on Automatic Control, 60(12), 3310-3315.

Lou, Y., Shi, G., Johansson, K.H., \& Hong, Y. (2014). Approximate Projected Consensus for Convex Intersection Computation: Convergence Analysis and Critical Error Angle. IEEE Transactions on Automatic Control, 59(7), 1722-1736.

Low, S. H., Paganini, F., \& Doyle, J. C. (2002). Internet congestion control. IEEE Control Systems magazine, 22(1), 28-43.

Mesbahi, M., \& Egerstedt, M. (2010). Graph Theoretic Methods for Multiagent Networks, Princeton: Princeton University Press.

Nagurney, A., \& Zhang, D. (1995). Projected dynamical systems and variational inequalities with applications (Vol. 2). Springer Science \& Business Media.

Necoara, I., Nesterov, Y., \& Glineur, F. (2011). A random coordinate descent method on large optimization problems with linear constraints. Technical Report, University Politehnica Bucharest, Romanian.

Nedic, A., Ozdaglar, A., \& Parrilo, A. P.(2010). Constrained Consensus and Optimization in Multi-Agent Networks. IEEE Transactions on Automatic Control, 55(4), 922-938.

Qiu, Z., Liu, S., \& Xie, L.(2016). Distributed constrained optimal consensus of multi-agent systems. Automatica, 68, 209-215.

Ruszczynski, A. P. (2006). Nonlinear Optimization, Princeton: Princeton university press.

Sayed, A. H. (2014). Adaptation, learning, and optimization over networks. Foundations and Trends in Machine Learning, 7(4-5), 311-801.

Shi, G., Johansson, K. H., \& Hong, Y. (2013). Reaching an optimal consensus: dynamical systems that compute intersections of convex sets. IEEE Transactions on Automatic Control, 58(3), 610-622.

Venets, V. I. (1985). Continuous algorithms for solution of convex optimization problems and finding saddle points of contex-coneave functions with the use of projection operations. Optimization, 16(4), 519-533.

Wang, J., \& Elia, N.(2011). A control perspective for centralized and distributed convex optimization. Proceding of IEEE Conference on Decision and Control, Orlando, USA (pp. 3800-3805)

Xia, S., \& Wang, J. (2000). On the stability of globally projected dynamical systems. Journal of Optimization Theory and Applications, 106(1), 129-150.

Xiao, L., \& Boyd, S. (2006). Optimal Scaling of a gradient method for distributed resource allocation. Journal of Optimization Theory and Applications, 129(3), 469-488.

Yi, P. \& Hong, Y. (2014). Quantized Subgradient Algorithm and Data-Rate 
Analysis for Distributed Optimization. IEEE Transactions on Control of Network Systems, 1(4), 380-392.

Yi, P., Hong, Y., \& Liu, F. (2015). Distributed Gradient Algorithm for Constrained Optimization with Application to Load Sharing in Power Systems. Systems $\mathcal{E}$ Control Letter, 83, 45-52.

Zhang, W., Liu, W., Wang, X., Liu, L., \& Ferrese, F. (2015). Online Optimal Generation Control Based on Constrained Distributed Gradient Algorithm. IEEE Transactions on Power Systems, 30(1), 35-45.

Zhao, C., Topcu, U., Li, N., \& Low, S. H. (2014). Design and stability of load-side primary frequency control in power systems. IEEE Transactions on Automatic Control, 59(5), 1177-1189. 